\title{
Fluctuation-dissipation in active matter
}

Cite as: J. Chem. Phys. 150, 184901 (2019); https://doi.org/10.1063/1.5081725

Submitted: 15 November 2018 . Accepted: 21 April 2019 . Published Online: 09 May 2019

Eric W. Burkholder (D), and John F. Brady (D)
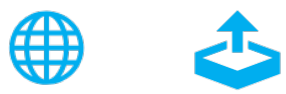

Export Citation

\section{ARTICLES YOU MAY BE INTERESTED IN}

Hydrodynamic interactions dominate the structure of active swimmers' pair distribution functions

The Journal of Chemical Physics 150, 184902 (2019); https://doi.org/10.1063/1.5085755

Dynamics in reactions on metal surfaces: A theoretical perspective

The Journal of Chemical Physics 150, 180901 (2019); https://doi.org/10.1063/1.5096869

Stochastic modeling of macromolecules in solution. I. Relaxation processes

The Journal of Chemical Physics 150, 184107 (2019); https://doi.org/10.1063/1.5077065

The Journal 


\title{
Fluctuation-dissipation in active matter
}

\author{
Cite as: J. Chem. Phys. 150, 184901 (2019); doi: 10.1063/1.5081725 \\ Submitted: 15 November 2018 • Accepted: 21 April 2019 • \\ Published Online: 9 May 2019
}

\author{
Eric W. Burkholder ${ }^{a)}$ (D) and John F. Brady
}

\author{
AFFILIATIONS \\ Division of Chemistry and Chemical Engineering, California Institute of Technology, Pasadena, California 91125, USA \\ Note: This article is part of the Special Topic "Chemical Physics of Active Matter" in J. Chem. Phys. \\ a) Now at Department of Physics, Stanford University, Stanford, California 94305, USA.

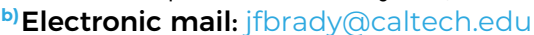

\begin{abstract}
In a colloidal suspension at equilibrium, the diffusive motion of a tracer particle due to random thermal fluctuations from the solvent is related to the particle's response to an applied external force, provided this force is weak compared to the thermal restoring forces in the solvent. This is known as the fluctuation-dissipation theorem (FDT) and is expressed via the Stokes-Einstein-Sutherland (SES) relation $D=k_{B} T / \zeta$, where $D$ is the particle's self-diffusivity (fluctuation), $\zeta$ is the drag on the particle (dissipation), and $k_{B} T$ is the thermal Boltzmann energy. Active suspensions are widely studied precisely because they are far from equilibrium-they can generate significant nonthermal internal stresses, which can break the detailed balance and time-reversal symmetry-and thus cannot be assumed to obey the FDT $a$ priori. We derive a general relationship between diffusivity and mobility in generic colloidal suspensions (not restricted to near equilibrium) using generalized Taylor dispersion theory and derive specific conditions on particle motion required for the FDT to hold. Even in the simplest system of active Brownian particles (ABPs), these conditions may not be satisfied. Nevertheless, it is still possible to quantify deviations from the FDT and express them in terms of an effective SES relation that accounts for the ABPs conversion of chemical into kinetic energy.
\end{abstract}

Published under license by AIP Publishing. https://doi.org/10.1063/1.5081725

\section{INTRODUCTION}

The fluctuation-dissipation theorem (FDT) states "a general relationship between the response of a given system to an external disturbance and the internal fluctuations of the system in the absence of the disturbance." This is true across a variety of systems, whether the fluctuations be thermal (classical), e.g., JohnsonNyquist noise in a resistor, or quantized such as the fluctuations of an electric field in a vacuum. The key assumptions of the FDT are that the system is in equilibrium in the absence of the disturbancethe probability distribution of states is Boltzmann-distributed with respect to the Hamiltonian of the system-and that the external disturbance is sufficiently weak that the tenets of linear response theory apply.

One of the most well-known manifestations of the FDT is the Brownian motion of a tracer particle suspended in a fluid. At thermal equilibrium, the motion of the tracer particle fluctuates due to collisions with the molecules of the solvent, giving rise to Brownian motion. The average velocity and displacement of the particle are zero, and the tracer motion is characterized by its mean-squared displacement (MSD), which grows linearly with time; the time derivative of the MSD gives the self-diffusivity $\boldsymbol{D}$. If one applies a weak external force $\boldsymbol{F}^{\text {ext }}$ to the tracer particle and drags it through the fluid, the velocity of the particle $\boldsymbol{U}$ will be linear in the applied force: $\boldsymbol{U}=\boldsymbol{M}^{U F} \cdot \boldsymbol{F}^{\text {ext }}$, where $\boldsymbol{M}^{U F}$ is the hydrodynamic mobility of the particle-the inverse of the drag on the particle. (The superscript UF denotes the hydrodynamic coupling between the velocity and force.) The FDT manifests in the Stokes-Einstein-Sutherland (SES) relation $\boldsymbol{D}=k_{B} T M^{U F}$, where $k_{B}$ is Boltzmann's constant and $T$ is the temperature of the system. (We will refer to the quantity $k_{B} T$ as the temperature in this article, as $k_{B}$ and $T$ will never appear independently of one another.) Alternatively expressed, the product of the self-diffusivity (fluctuation) and drag (dissipation) is precisely equal to the system temperature $\boldsymbol{D} \cdot\left(\boldsymbol{M}^{U F}\right)^{-1}=k_{B} T \boldsymbol{I}$ (where $\boldsymbol{I}$ is the isotropic tensor).

This theorem does not require us to say anything about the probe particle or the medium through which it moves-the relationship between the drag and the self-diffusivity is specified only by the temperature of the system. Indeed, if we instead considered the motion of a tracer particle in a suspension of other particles (see Fig. 1), this relation would be true regardless of any interactions among the particles, the concentrations of other species, or the spatial distribution of the particles (the microstructure). While both $\boldsymbol{D}$ and $\left(\boldsymbol{M}^{U F}\right)^{-1}$ are dependent on suspension 


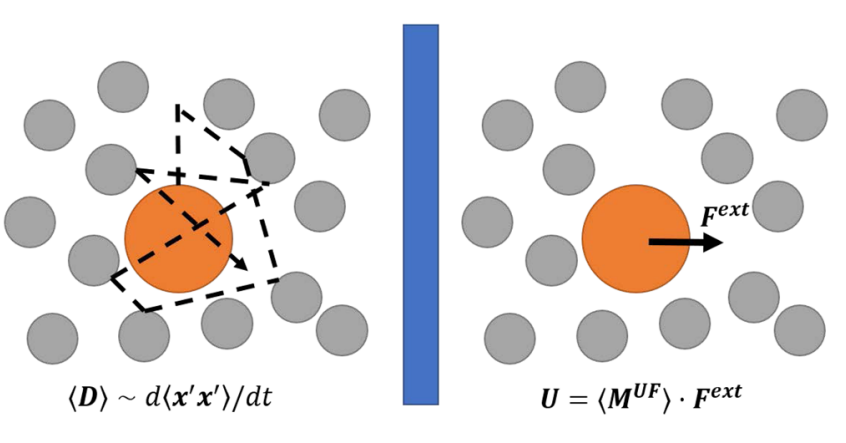

$\langle\boldsymbol{D}\rangle \cdot\left\langle\boldsymbol{M}^{\boldsymbol{U} \boldsymbol{F}}\right\rangle^{-\mathbf{1}}=k_{B} T \boldsymbol{I}$

FIG. 1. Sketch of the fluctuation-dissipation theorem (FDT) in a colloidal suspension. Left: A tracer particle diffuses in a suspension of bath particles due to random Brownian motion. The time derivative of the mean squared-displacements due to Brownian motion $\left\langle\boldsymbol{x}^{\prime} \boldsymbol{x}^{\prime}\right\rangle$ is proportional to the self-diffusivity of the particle $\langle\boldsymbol{D}\rangle$. Right: The same tracer particle moves through the same suspension under the action of an external force $\boldsymbol{F}^{\text {ext }}$. The speed of this particle is linear in the external force, with the constant of proportionality being the average mobility $\left\langle\boldsymbol{M}^{\mathrm{UF}}\right\rangle$. The FDT states that these two problems are fundamentally related by $\langle\boldsymbol{D}\rangle \cdot\left\langle\boldsymbol{M}^{U F}\right\rangle^{-1}=k_{B} T \boldsymbol{I}$, where $k_{B} T$ is the temperature of the system and is independent of all other suspension properties (composition, interparticle interactions, etc.)

properties, their product is always equal to the temperature of the system and nothing else. This is true on all time scales long compared to the momentum relaxation time of the particle, when the tracer motion is diffusive. ${ }^{4}$ Thus, for systems that obey the FDT, one can do a single experiment-tracking the displacements of the tracer particle to compute $\boldsymbol{D}$ or applying a weak external force to the tracer and measuring its average speed to infer $\boldsymbol{M}^{U F}$ - to characterize both the internal fluctuations of the system and the response to an external stimulus at a given temperature.

Active systems have recently generated substantial interest among soft-matter and statistical physicists. The constituents of these systems are able to self-propel through some physicochemical mechanism, which generates internal stresses that drive the system out of equilibrium without the influence of external forces. One cannot assume $a$ priori that the FDT may be applied because the steady-state internal fluctuations are not an equilibrium process. Indeed, it is not even clear how one might define the chemical potential, free energy, or temperature for active systems-a challenge which has fueled a spirited debate in the literature. ${ }^{7-}$ Any attempts have concluded that an effective temperature depends not only on the particle activity but also on suspension properties such as concentration and composition. Despite this complication, experimental and theoretical predictions of enhanced tracer diffusion ${ }^{10-18}$ and reduced shear viscosity and microviscosity in active systems ${ }^{19-24}$ are at least qualitatively consistent with the FDT.

To illustrate this, consider as a minimal model of active systems a single active Brownian particle (ABP) of size $a$ which "swims" at some constant speed $U_{0}$ in a direction $\boldsymbol{q}$ that changes randomly over a time scale $\tau_{R}$. At times long compared to $\tau_{R}$, the ABP diffuses with a long-time "active" self-diffusivity $D^{a c t}=D_{T}+D^{\text {swim }}$, where $D_{T}$ is its thermal Stokes-Einstein-Sutherland (SES) diffusivity and the purely mechanical, isotropic "swim" diffusivity is $D^{\text {swim }}=U_{0}^{2} \tau_{R} / 6$ (in 3-D). The drag on an ABP in a Newtonian solvent is given by its usual translational Stokes drag $\zeta_{s}$. In the spirit of the SES relation and $\mathrm{FDT}$, we can write $\zeta_{s} D^{a c t}=k_{B} T+k_{s} T_{s}$, where $k_{s} T_{s} \equiv \zeta_{s} D^{\text {swim }}$ defines the "activity" of the particle. For a single active particle, we thus have a generalized SES relation that states that the product of the drag and the active self-diffusivity is equal to the thermal energy of the solvent, plus the "kinetic energy" of the swimmer. Note that we have not specified anything about the swimming mechanism, only that the particle undergoes an active random walk.

This simple single-particle result shows promising similarity with classical manifestations of the FDT. But how general is it? It only shows that if one uses an active particle as a tracer in a solvent, the SES is modified by the addition of "internal" active fluctuations of the tracer itself. But what if one were to instead place a passive tracer particle in a fluid that also contained a dispersion of active particles? Is the relation between the fluctuations of the tracer particle and the drag as it moves through the suspension still linear in $k_{B} T+k_{s} T_{s}$ ? Does the fluctuation-dissipation relation depend on the activity only, or also on the volume fraction, etc., of the swimmers? Both the active or long-time self-diffusivity (LTSD) of a tracer in an active bath, as well as the "temperature" of an active bath, have been shown to depend on properties of the system such as particle size, swimmer fraction, swimming mechanism, etc. ${ }^{7,8,10,11,13,15,17,25-28}$ At present, there are few measurements of the self-drag on a tracer in an active suspension. ${ }^{23,25,29,30}$ Both the drag and the diffusivity are needed to characterize the fluctuation-dissipation relation-and departures from it-in an active suspension.

It should be appreciated that for active systems to even approximately obey the FDT is remarkable. In a passive system, the diffusivity of a particle decreases with increasing suspension concentration due to the crowding effect of other particles (see Fig. 1). The particle's drag, on the other hand, increases from this same crowding effect. Their product is the constant temperature. But just the opposite must occur in an active bath: the diffusivity of a tracer particle is enhanced by activity, while its drag must, therefore, decrease with activity. How and why the drag decreases, and the validity of the FDT, is the subject of this study. As we show, determining the drag is equivalent to the microrheology problem and thus our results are more broadly applicable than just the FDT.

In this article, we use generalized Taylor dispersion theory to make a direct connection between fluctuation and dissipation, deriving expressions for the average velocity (and thus, drag) and LTSD of a tracer (probe) particle in a generic colloidal suspension. The complete derivations for all suspension concentrations, bath particle activities, or any other nonequilibrium effects (e.g., external shear flow) are given in Appendixes A and B.

To illustrate the general behavior, consider a dilute suspension where only pairwise interactions between the tracer particle (1), and a single bath particle (2) are considered-this is depicted in Fig. 2. (The condition for diluteness is that the volume fraction $\phi$ based on the bath particle size is small $\phi \ll 1$.) In the absence of external forces, the average diffusivity $\langle\boldsymbol{D}\rangle$ of the probe in this dilute suspension is 




FIG. 2. Schematic of the model system: a Brownian probe particle of size $R$ immersed in a suspension of $A B P s$ with size $a$ at number density $n^{\infty}$-the centerto-center separation distance upon a collision is denoted by $R_{c}=R+a$. The ABPs swim in a direction $\boldsymbol{q}$ at speed $U_{0} ; \boldsymbol{q}$ changes randomly on a time scale characterized by $\tau_{R}$. The probe translates under the action of a constant external force $F^{\text {ext }}$.

$$
\begin{aligned}
\langle\boldsymbol{D}\rangle= & \int \boldsymbol{D}_{11} \hat{P}_{1 / 1}^{(0,0)} d \boldsymbol{r}-\int \boldsymbol{U}_{\mathbf{1}}^{(\mathbf{0})} \hat{\boldsymbol{d}}^{(\boldsymbol{k})} d \boldsymbol{r} \\
& +\int\left(\boldsymbol{D}_{12}-\boldsymbol{D}_{11}\right) \cdot \nabla_{r} \hat{\boldsymbol{d}}^{(\boldsymbol{k})} d \boldsymbol{r}
\end{aligned}
$$

while the average mobility (inverse of the drag) in response to a weak external force is

$$
\begin{aligned}
\left\langle\boldsymbol{M}^{U F}\right\rangle= & \int \boldsymbol{M}_{11}^{U F} \hat{P}_{1 / 1}^{(0,0)} d \boldsymbol{r}-\int \boldsymbol{U}_{1}^{(0)} \hat{\boldsymbol{d}}^{(F)} d \boldsymbol{r} \\
& +\int\left(\boldsymbol{D}_{12}-\boldsymbol{D}_{11}\right) \cdot \nabla_{r} \hat{\boldsymbol{d}}^{(F)} d \boldsymbol{r}
\end{aligned}
$$

In these expressions, $\hat{P}_{1 / 1}^{(0,0)}(\boldsymbol{r})$ is the probability of finding the bath particle at some position $\boldsymbol{r}$ relative to the probe in the absence of any forcing. (Here, in this section, $\boldsymbol{r}$ is a short-hand for both the position and orientation of the bath particle relative to the probe.) The diffusivity tensors, $D_{11}=k_{B} T M_{11}^{U F}$ and $D_{12}=k_{B} T M_{12}^{U F}$, are given by the well-known hydrodynamic self- and pair-mobility of the probe particle, respectively. ${ }^{31}$ The velocity of the probe particle due to activity, interparticle forces, external shear, etc., is $\boldsymbol{U}_{1}^{(0)}$; this velocity excludes only the velocities due to the external force $\boldsymbol{M}_{11}^{U F} \cdot \boldsymbol{F}^{\text {ext }}$ and Brownian motion. The long-wavelength fluctuations in the probe's position due to Brownian forces in the solvent are described by the displacement field $\hat{\boldsymbol{d}}^{(k)}$, and its fluctuations due to the action of the external force are given by $\hat{\boldsymbol{d}}^{(F)}$.

Equations (1) and (2) show that the diffusivity and mobility are formally identical (modulo a factor of $k_{B} T$ ) under the lens of generalized Taylor dispersion theory. For the FDT to hold, we only require that $\hat{\boldsymbol{d}}^{(\boldsymbol{k})}=k_{B} T \hat{\boldsymbol{d}}^{(F)}$. The motion due to the fluctuating Brownian force $-i k k_{B} T$ must be mechanically identical to that from the external force $\boldsymbol{F}^{\text {ext }}$; this is the mechanical underpinning of the FDT for systems in thermodynamic equilibrium.
Fluctuations arising from the thermal forces in the solvent are described by (see Appendixes A and B)

$$
\begin{aligned}
\frac{\partial \hat{\boldsymbol{d}}^{(\boldsymbol{k})}}{\partial t} & +\nabla_{r} \cdot\left(\left[\boldsymbol{U}_{\mathbf{2}}^{(\mathbf{0})}-\boldsymbol{U}_{\mathbf{1}}^{(\mathbf{0})}\right] \hat{\boldsymbol{d}}^{(\boldsymbol{k})}-\boldsymbol{D}^{r e l} \cdot \nabla_{r} \hat{\boldsymbol{d}}^{(\boldsymbol{k})}\right) \\
= & \hat{P}_{1 / 1}^{(0,0)}\left(\left\langle\boldsymbol{U}^{\text {probe }}\right\rangle-\boldsymbol{U}_{\mathbf{1}}^{(\mathbf{0})}\right)+2\left(\boldsymbol{D}_{\mathbf{1 2}}-\boldsymbol{D}_{\mathbf{1 1}}\right) \cdot \nabla_{r} \hat{P}_{1 / 1}^{(0,0)} \\
& +\hat{P}_{1 / 1}^{(0,0)} \nabla_{r} \cdot\left(\boldsymbol{D}_{\mathbf{1 2}}-\boldsymbol{D}_{\mathbf{1 1}}\right),
\end{aligned}
$$

while those from the external force are described by

$$
\begin{aligned}
& \frac{\partial \hat{\boldsymbol{d}}^{(F)}}{\partial t}+\nabla_{r} \cdot\left(\left[\boldsymbol{U}_{\mathbf{2}}^{(\mathbf{0})}-\boldsymbol{U}_{\mathbf{1}}^{(\mathbf{0})}\right] \hat{\boldsymbol{d}}^{(F)}-\boldsymbol{D}^{r e l} \cdot \nabla_{r} \hat{\boldsymbol{d}}^{(F)}\right) \\
& =\nabla_{r} \cdot\left[\left(\boldsymbol{M}_{\mathbf{1 2}}^{U F}-\boldsymbol{M}_{\mathbf{1 1}}^{U F}\right) \hat{P}_{1 / 1}^{(0,0)}\right] .
\end{aligned}
$$

The quantity $D^{r e l}=D_{11}+D_{22}-D_{21}-D_{12}$ is the relative hydrodynamic mobility multiplied by $k_{B} T$.

The two fluctuations are the same only when the local probe velocity in the absence of the external force

$$
\boldsymbol{U}^{\text {probe }}=\boldsymbol{U}_{\mathbf{1}}^{(\mathbf{0})}-\left(\boldsymbol{D}_{\mathbf{1 2}}-\boldsymbol{D}_{11}\right) \cdot \nabla_{r} \ln \hat{P}_{1 / 1}^{(0,0)}
$$

is equal to its suspension average over $\hat{P}_{1 / 1}^{(0,0)}$

$$
\left\langle\boldsymbol{U}^{\text {probe }}\right\rangle=\int \boldsymbol{U}_{\mathbf{1}}^{(\mathbf{0})} \hat{P}_{1 / 1}^{(0,0)} d \boldsymbol{r}-\int\left(\boldsymbol{D}_{\mathbf{1 2}}-\boldsymbol{D}_{\mathbf{1 1}}\right) \cdot \nabla_{r} \hat{P}_{1 / 1}^{(0,0)} d \boldsymbol{r} .
$$

In general, the detailed and average probe velocities are not the same. They are the same, and the FDT is satisfied for a passive equilibrium suspension. In equilibrium, the velocity $\boldsymbol{U}_{\mathbf{1}}^{(\mathbf{0})}=-\left(\boldsymbol{M}_{\mathbf{1 2}}-\boldsymbol{M}_{\mathbf{1 1}}\right) \cdot \nabla_{r} V^{P}(\boldsymbol{r})$, where $V^{P}(\boldsymbol{r})$ is the interparticle potential, and the pair-probability is Boltzmann: $\hat{P}_{1 / 1}^{(0,0)} \sim \exp \left(-V^{P}(\boldsymbol{r}) / k_{B} T\right)$, and so $\boldsymbol{U}^{\text {probe }}=0$, as does its average. This was the case in the work of Zia and Brady.

In Sec. II, we show that this condition is not met in general for a dilute active suspension and therefore the product $\langle D\rangle \cdot\left\langle M^{U F}\right\rangle^{-1}$ depends on the geometry and activity of the suspension. However, in certain limits, we can recover the same fluctuation-dissipation relation as found for a single active particle moving in a Newtonian fluid.

\section{MODEL SYSTEM}

Consider the motion of a tracer particle of size $R$ with StokesEinstein-Sutherland (SES) diffusivity $D_{P}$ in an active suspension. In addition to Brownian fluctuations from the solvent, the tracer moves under the action of a constant external force $F^{\text {ext }}$. The suspension is composed of ABPs with size $a$, (constant) swim speed $U_{0}$, SES diffusivity $D_{T}$, and a characteristic reorientation time $\tau_{R}$ in a Newtonian solvent of viscosity $\eta_{s}$. For simplicity, we neglect hydrodynamic interactions (HI) and assume the suspension to be sufficiently dilute $\left(\phi=4 \pi n^{\infty} a^{3} / 3 \ll 1\right.$, where $n^{\infty}$ is the constant volumetric number density of swimmers far from the probe) that we only need to 
consider pairwise particle interactions. The dynamics of this system are described by the following Smoluchowski equation:

$$
\frac{\partial P_{2}}{\partial t}+\nabla_{z} \cdot \boldsymbol{j}_{P}^{T}+\nabla_{r} \cdot\left(\boldsymbol{j}_{s}^{T}-\boldsymbol{j}_{\boldsymbol{P}}^{T}\right)+\nabla_{R} \cdot \boldsymbol{j}_{s}^{\boldsymbol{R}}=0,
$$

where $\nabla_{R}=\boldsymbol{q} \times \nabla_{\boldsymbol{q}}$ is the orientation-space gradient operator for an axisymmetric particle. This is a conservation statement for the probability $P_{2}(\boldsymbol{z}, \boldsymbol{r}, \boldsymbol{q}, t)$ of finding a swimmer with position $\boldsymbol{r}$ and orientation $\boldsymbol{q}$ relative to a probe at position $\boldsymbol{z}$ (we neglect orientational degrees of freedom for the probe). The translational flux of the probe is $\boldsymbol{j}_{\boldsymbol{P}}^{\boldsymbol{T}}=\boldsymbol{M}^{\boldsymbol{U F}} \cdot \boldsymbol{F}^{\boldsymbol{e x t}} P_{2}-D_{P} \nabla_{z} P_{2}+D_{P} \nabla_{r} P_{2} ; \boldsymbol{M}^{U \boldsymbol{F}}=\boldsymbol{I} / \zeta_{P}$ is the bare probe mobility in the absence of $\mathrm{HI}$ and $\zeta_{P}$ is its (Stokes) drag coefficient. The translational flux of the swimmer is $\boldsymbol{j}_{\boldsymbol{s}}^{T}=U_{0} \boldsymbol{q} P_{2}-D_{T} \nabla_{r} P_{2}$ and its rotational flux is $j_{s}^{R}=-\tau_{R}^{-1} \nabla_{R} P_{2}$. The boundary conditions for the Smoluchowski equation are no flux at contact between the probe and an active bath particle and a uniform random distribution of bath particles far from the probe.

The average motion of the probe is governed by the singleparticle equation

$$
\frac{\partial \hat{P}_{1}}{\partial t}+i \boldsymbol{k} \cdot\left\langle\hat{\boldsymbol{j}}_{P}^{T}\right\rangle=0
$$

where the angle brackets represent an average over the swimmer configurations-over $d \boldsymbol{r} d \boldsymbol{q}$ - and we have taken a Fourier transform with respect to $z$. The average flux of the probe is

$$
\left\langle\hat{\boldsymbol{j}}_{\boldsymbol{P}}^{T}\right\rangle=\hat{P}_{1}\left[\boldsymbol{M}^{U F} \cdot \boldsymbol{F}^{\text {ext }}-i \boldsymbol{k} D_{P}+D_{P} \iint \nabla_{r} \hat{P}_{1 / 1} d \boldsymbol{r} d \boldsymbol{q}\right],
$$

where $\hat{P}_{2}(\boldsymbol{k}, \boldsymbol{r}, \boldsymbol{q}, t)=\hat{P}_{1 / 1}(\boldsymbol{k}, \boldsymbol{r}, \boldsymbol{q}, t) \hat{P}_{1}(\boldsymbol{k}, t)$. The long-time selfdiffusivity of the probe is given by the long-wavelength (small k) fluctuations in the conditional probability $\hat{P}_{1 / 1}$ so we write $\hat{P}_{1 / 1}=\hat{P}_{1 / 1}^{(0)}+i \boldsymbol{k} \cdot \hat{\boldsymbol{d}}^{(\boldsymbol{k})}+O\left(k^{2}\right)$ as in previous works. ${ }^{28,32,33}$ Expanding the average probe flux with respect to $\boldsymbol{k}\left(\left\langle\hat{\boldsymbol{j}}_{\boldsymbol{P}}^{T}\right\rangle=\hat{P}_{1}\left[\left\langle\boldsymbol{U}^{\text {probe }}\right\rangle-i \boldsymbol{k} \cdot\langle\boldsymbol{D}\rangle+\ldots\right]\right)$ defines the average velocity and long-time self-diffusivity (LTSD)

$$
\begin{gathered}
\left\langle\boldsymbol{U}^{\text {probe }}\right\rangle \equiv \boldsymbol{M}^{U \boldsymbol{U}} \cdot \boldsymbol{F}^{\boldsymbol{e x t}}+D_{P} \int \nabla_{r} \hat{P}_{1 / 1}^{(0)} d \boldsymbol{r} d \boldsymbol{q}, \\
\langle\boldsymbol{D}\rangle \equiv D_{P}\left[\boldsymbol{I}-\int \nabla_{r} \hat{\boldsymbol{d}}^{(\boldsymbol{k})} d \boldsymbol{r} d \boldsymbol{q}\right] .
\end{gathered}
$$

When the external force is weak compared to Brownian motion, we can also expand the microstructure with respect to $\boldsymbol{F}^{\text {ext }}: \hat{P}_{1 / 1}^{(0)}=\hat{P}_{1 / 1}^{(0,0)}-\boldsymbol{F}^{e x t} \cdot \hat{\boldsymbol{d}}^{(F)}+O\left(\left(F^{e x t}\right)^{2}\right)$. The average speed of the probe is now

$$
\left\langle\boldsymbol{U}^{\text {probe }}\right\rangle=\underbrace{D_{P} \int \nabla_{r} \hat{P}_{1 / 1}^{(0,0)} d \boldsymbol{r} d \boldsymbol{q}}_{\left\langle\boldsymbol{U}^{\text {drift }}\right\rangle}+\boldsymbol{F}^{\text {ext }} \cdot \underbrace{\left[\boldsymbol{M}^{\boldsymbol{U F}}-D_{P} \int \nabla_{r} \hat{\boldsymbol{d}}^{(F)} d \boldsymbol{r} d \boldsymbol{q}\right]}_{\left\langle M^{U F}\right\rangle} .
$$

The first term in the above equation is the probe's drift velocity-the average velocity resulting from nonzero suspensionaveraged forces acting on the probe (other than the external force). For a passive thermal system, there is no net motion of any particle at equilibrium regardless of the particle shape. For an active system, however, spontaneous motion of a nonspherical probe particle can arise when the run length of the active bath particles, $U_{0} \tau_{R}$, is comparable to the radius of curvature of the probe particle; ${ }^{34-36}$ this spontaneous motion is captured by this drift velocity. This is most easily seen by using the divergence theorem to write

$$
\left\langle\boldsymbol{U}^{d r i f t}\right\rangle=-D_{P} \oint \boldsymbol{n} n_{0} d S
$$

where $\boldsymbol{n}$ is the outer normal to the probe surface and $n_{0}=\int \hat{P}_{1 / 1}^{(0,0)} d \boldsymbol{q}$ is the local number density of bath particles at contact [see (14) below] where the no-flux condition is imposed for hard particles. For active bath particles $n_{0}$ can be nonuniform over the surface of contact resulting in a net force and motion of the probe particle.

The expression (12) for $\left\langle\boldsymbol{M}^{U F}\right\rangle$ is formally identical to (11) for $\langle\boldsymbol{D}\rangle$-the LTSD of the probe is the same as its mobility under the action of a small external force (modulo a factor of $k_{B} T$ ). Although the formal relations are the same, the mobility of the particle is not identical to its self-diffusivity unless $\hat{\boldsymbol{d}}^{(\boldsymbol{k})}=k_{B} T \hat{\boldsymbol{d}}^{(F)}$. In the introduction (and more generally in Appendix B), we showed that the two fluctuation fields are not governed by the same equations for a generic suspension not in equilibrium. For the model suspension considered here, the FDT is not satisfied unless $\nabla_{r} \ln \hat{P}_{1 / 1}^{(0,0)}=0$. For passive hard particles, $\hat{P}_{1 / 1}^{(0,0)}$ is constant everywhere and the FDT is obeyed. However, active particles accumulate at no-flux surfaces, ${ }^{37,38} \hat{P}_{1 / 1}^{(0,0)}$ is not constant, and thus the FDT is not obeyed in general.

For a single ABP, there are no accumulation no-flux surfaces, and thus, a modified FDT is possible: the product of the active diffusivity and the drag, $\zeta_{s} D^{a c t}=k_{B} T+k_{s} T_{s}$, is linear in the "temperature." An analogous statement for the passive tracer in an active suspension would thus be $\left\langle\boldsymbol{M}^{\boldsymbol{U F}}\right\rangle^{-1} \cdot\langle\boldsymbol{D}\rangle=k_{B} T+E^{\text {act }}$, where the "active energy" $E^{\text {act }}$ only depends on the activity, $k_{s} T_{s}$, of the bath particles. It is the purpose of this paper to investigate under what conditions such a modified FDT might hold.

To determine the generalized SES relation for active suspensions, we thus need to solve for $\langle\boldsymbol{D}\rangle$ and then separately for $\left\langle\boldsymbol{M}^{U F}\right\rangle$ (or $E^{a c t}$ ). In a previous paper, Burkholder and Brady, ${ }^{28}$ we determined the diffusivity of a tracer in a dilute active bath (see Sec. III B). In this paper, we solve the problem for the average mobility (or average drag) and thus can assess the validity of the FDT. Determining the average drag is precisely the fixed-force microrheology problem for a probe in an active bath, and thus, our results have applications beyond the present discussion.

We solve the Smoluchowski Eq. (7) using the familiar method popularized by Saintillan and Shelley ${ }^{39}$ and expand the pairdistribution function in terms of orthogonal tensor harmonics in $\boldsymbol{q}$

$$
\begin{aligned}
& \hat{P}_{1 / 1}^{(0,0)}(\boldsymbol{r}, \boldsymbol{q} ; t)=n_{0}(\boldsymbol{r} ; t)+\boldsymbol{q} \cdot \boldsymbol{m}_{\mathbf{0}}(\boldsymbol{r} ; t) \\
& \quad+\left(\boldsymbol{q} \boldsymbol{q}-\frac{\boldsymbol{I}}{3}\right): \boldsymbol{Q}_{\mathbf{0}}(\boldsymbol{r} ; t)+\left(\boldsymbol{q} \boldsymbol{q} \boldsymbol{q}-\frac{\boldsymbol{\alpha} \cdot \boldsymbol{q}}{5}\right) \odot \boldsymbol{B}_{\mathbf{0}}+\ldots,
\end{aligned}
$$




$$
\begin{aligned}
\hat{\boldsymbol{u}} \cdot \hat{\boldsymbol{d}}^{(F)}(\boldsymbol{r}, \boldsymbol{q} ; t)=n^{\prime}(\boldsymbol{r} ; t)+\boldsymbol{q} \cdot \boldsymbol{m}^{\prime}(\boldsymbol{r} ; t) \\
\quad+\left(\boldsymbol{q} \boldsymbol{q}-\frac{\boldsymbol{I}}{3}\right): \boldsymbol{Q}^{\prime}(\boldsymbol{r} ; t)+\left(\boldsymbol{q} \boldsymbol{q q}-\frac{\boldsymbol{\alpha} \cdot \boldsymbol{q}}{5}\right) \odot \boldsymbol{B}^{\prime}+\ldots
\end{aligned}
$$

where $\boldsymbol{I}$ is the identity tensor, and $\boldsymbol{\alpha}$ is the fourth-order isotropic tensor. The unit vector in the direction of the external force is $\hat{\boldsymbol{u}}$. The zeroth moment $n$ is the concentration field, $\boldsymbol{m}$ is the polar order, $\boldsymbol{Q}$ is the nematic order, and so on. ${ }^{39,40}$ In the absence of external forces, the closure $\boldsymbol{Q}_{\mathbf{0}}=\mathbf{0}$ results in qualitative and often good quantitative agreement between calculations of the particle-phase pressure using theory and Brownian dynamics simulations-the closure $\boldsymbol{B}_{\mathbf{0}}=0$ provided nearly exact quantitative agreement between theory and simulation. ${ }^{37}$ We examine both closures in this problem and provide a comparison to the exact solution in 2D in Appendix D.

The governing equations for $n_{0}, \boldsymbol{m}_{\mathbf{0}}$ and $\boldsymbol{Q}_{\mathbf{0}}$ may be found in the appendix of Yan and Brady. ${ }^{37}$ Applying the moments-averaging procedure to the governing equation and boundary conditions for $\hat{\boldsymbol{u}} \cdot \hat{\boldsymbol{d}}^{(F)}$ yields a system of coupled PDEs governing the steady-state perturbed microstructure

$$
\begin{gathered}
\nabla_{r} \cdot\left[U_{0} \boldsymbol{m}^{\prime}-D^{r e l} \nabla n^{\prime}\right]=\boldsymbol{M}^{U F} \cdot \boldsymbol{F}^{e x t} \cdot \nabla n_{0} \\
\nabla_{r} \cdot\left[U_{0}\left(\boldsymbol{I} \frac{n^{\prime}}{3}+\boldsymbol{Q}^{\prime}\right)-D^{r e l} \nabla \boldsymbol{m}^{\prime}\right]+2 D_{R} \boldsymbol{m}^{\prime}=\boldsymbol{M}^{U F} \cdot \boldsymbol{F}^{e x t} \cdot \nabla \boldsymbol{m}_{\mathbf{0}} \\
\nabla_{r} \cdot\left[U_{0}\left(\frac{\boldsymbol{\alpha} \cdot \boldsymbol{m}^{\prime}}{5}-\boldsymbol{I} \frac{\boldsymbol{m}^{\prime}}{3}\right)-D^{r e l} \nabla \boldsymbol{Q}^{\prime}\right]+6 D_{R} \boldsymbol{Q}^{\prime}=\boldsymbol{M}^{U F} \cdot \boldsymbol{F}^{\text {ext }} \cdot \nabla \boldsymbol{Q}_{0}
\end{gathered}
$$

where we have neglected terms of $O(i \boldsymbol{k})$, as they are not necessary to determine the average probe speed. The relative translational diffusivity is the sum of the bare probe and swimmer SES diffusivities $D^{r e l}=D_{P}+D_{T}$. Note that these governing equations are valid even when the external force is not weak-i.e., when the expansion $\hat{P}_{1 / 1}^{(0)}=\hat{P}_{1 / 1}^{(0,0)}-\boldsymbol{F}^{\text {ext }} \cdot \hat{\boldsymbol{d}}^{(F)}+O\left(\left(F^{\text {ext }}\right)^{2}\right)$ is no longer valid-and can thus be applied to the nonlinear microrheology problem (see Burkholder and Brady $^{41}$ ). flux

At particle contact $r=R_{c} \equiv R+a$, there can be no translational

$$
\begin{gathered}
\boldsymbol{n} \cdot\left[U_{0} \boldsymbol{m}^{\prime}-D^{r e l} \nabla n^{\prime}\right]=\boldsymbol{n} \cdot \boldsymbol{M}^{U F} \cdot \boldsymbol{F}^{e x t} n_{0} \\
\boldsymbol{n} \cdot\left[U_{0}\left(\boldsymbol{I} \frac{n^{\prime}}{3}+\boldsymbol{Q}^{\prime}\right)-D^{r e l} \nabla \boldsymbol{m}^{\prime}\right]=\boldsymbol{n} \cdot \boldsymbol{M}^{U F} \cdot \boldsymbol{F}^{\boldsymbol{e x t}} \boldsymbol{m}_{\mathbf{0}} \\
\boldsymbol{n} \cdot\left[U_{0}\left(\frac{\boldsymbol{\alpha} \cdot \boldsymbol{m}^{\prime}}{5}-\boldsymbol{I} \frac{\boldsymbol{m}^{\prime}}{3}\right)-D^{r e l} \nabla \boldsymbol{Q}^{\prime}\right]=\boldsymbol{n} \cdot \boldsymbol{M}^{U F} \cdot \boldsymbol{F}^{e x t} \boldsymbol{Q}_{0}
\end{gathered}
$$

where $\boldsymbol{n}$ is the outward-pointing unit normal of the probe. In the far-field, there is no long-ranged order $\hat{P}_{1 / 1}^{(0)}=n^{\infty} / 4 \pi$ as $r \rightarrow \infty$, and thus, the fluctuations are zero

$$
n^{\prime}, \boldsymbol{m}^{\prime}, \boldsymbol{Q}^{\prime} \sim 0, r \rightarrow \infty .
$$

One may solve this system analytically when $\boldsymbol{Q}^{\prime}=0$ (see Appendix C). For $\boldsymbol{Q}^{\prime} \neq 0$, the solution must be obtained numerically, which we do using a second-order finite difference scheme. ${ }^{42}$

\section{PROBE SPEED AND SELF-DRAG}

In the linear-response regime, the probe velocity is linear in the applied external force

$$
\left\langle\boldsymbol{U}^{\text {probe }}\right\rangle=\boldsymbol{F}^{\text {ext }} \cdot\left[\boldsymbol{M}^{U F}-D_{P} \int \nabla_{r} \hat{\boldsymbol{d}}^{(F)} d \boldsymbol{r} d \boldsymbol{q}\right] .
$$

Due to the symmetry of this problem, $\left\langle\boldsymbol{U}^{\text {drift }}\right\rangle$ is zero, and the probe mobility is isotropic, $\left\langle\boldsymbol{M}^{U F}\right\rangle=\boldsymbol{I}\langle\mu\rangle$, where $\langle\mu\rangle$ is the mobility coefficient. In general, the mobility problem (the velocity of a particle due to an applied force) and the resistance problem (the force on a particle moving at a particular speed) are different. For the simple case of the probe particle (1) and a single swimmer (2), the probe's hydrodynamic self-mobility is

$$
M_{11}^{U F}=\left[\boldsymbol{R}_{11}^{F U}-\boldsymbol{R}_{12}^{F U} \cdot\left(\boldsymbol{R}_{22}^{F U}\right)^{-1} \cdot \boldsymbol{R}_{21}^{F U}\right]^{-1} \neq\left(\boldsymbol{R}_{11}^{F U}\right)^{-1},
$$

where the linear relation between the velocity of particle $\alpha$ and the force on particle $\beta$ is $M_{\alpha \beta}^{U F}$, and the relation between the force on particle $\alpha$ due to the motion of particle $\beta$ is $R_{\alpha \beta}^{F U}$. The selfdrag of the probe $R_{11}^{F U}$ is not equal to the inverse of its selfmobility $\boldsymbol{M}_{\mathbf{1 1}}^{\boldsymbol{U F}}$, in general, ${ }^{31}$ rather it depends on the hydrodynamic interactions between all the other particles in the suspension. Even if the average hydrodynamic mobility and resistance are isotropic, $\left\langle\boldsymbol{M}_{11}^{U F}\right\rangle=\left\langle\mu^{H I}\right\rangle \boldsymbol{I}$ and $\left\langle\boldsymbol{R}_{11}^{F U}\right\rangle=\left\langle\zeta^{H I}\right\rangle \boldsymbol{I}$, the mobility and drag are not necessarily inverses $\left\langle\mu^{H I}\right\rangle \neq 1 /\left\langle\zeta^{H I}\right\rangle$. In the special case under consideration - where we neglect fluid velocity disturbances of the particles-the probe's hydrodynamic self-drag is just the Stokes drag $\zeta_{P}$ and the hydrodynamic self-drag and selfmobility are inverses. This allows us to easily move from the mobility formulation of generalized Taylor dispersion theory to the resistance formulation implicit in discussing the drag on the probe.

In addition to the hydrodynamic contribution to the mobility (and drag), there is the interparticle contribution represented by the integral in (23), which is the entropic restoring force in the bath. The total drag on the probe-the Stokes drag plus the additional drag due to interparticle interactions in the dilute bath-is given by

$$
\langle\zeta\rangle=\zeta_{P}\left[1+\frac{k_{B} T}{F^{e x t}} \int_{R_{c}} \hat{\boldsymbol{u}} \cdot \boldsymbol{n} n^{\prime} d S\right]
$$

which we have expressed as an integral of the concentration fluctuation $n^{\prime}$ over the contact surface $R_{c}$. Note that the interparticle contribution depends only on interactions at the no-flux boundary, whereas the hydrodynamic (and Brownian) contribution(s) will, in general, depend on the entire configuration of the suspension. The "undisturbed" concentration, $n_{0}$, while not constant owing to the accumulation of active particles at the probe surface [see (C6)], is spherically symmetric and does not contribute to the average drag.

Conventionally, the self-drag $\langle\zeta\rangle$ of the probe is expressed in terms of a suspension-averaged viscosity: $\langle\zeta\rangle=6 \pi\langle\eta\rangle R$, where $\langle\eta\rangle$ is known as the microviscosity of the suspension. ${ }^{42,43}$ In the absence of any hydrodynamic interactions or interparticle forces, the microviscosity of a passive suspension is $\langle\eta\rangle=\eta_{s}\left(1+\phi^{e x}\right)$, where the excluded volume fraction is $\phi^{e x}=\phi(1+R / a)^{2} / 2$. 


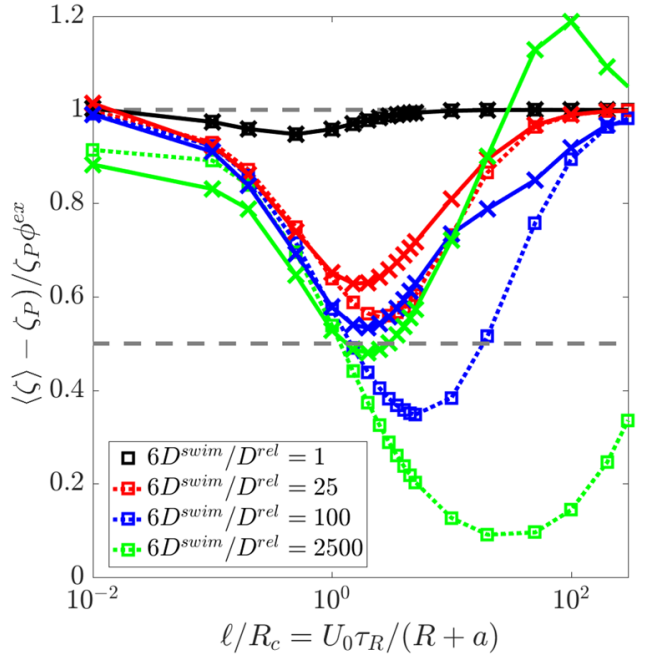

FIG. 3. Particle contribution to the probe drag scaled by its value in a passive suspension $\zeta_{P} \phi^{\text {ex }}$. The Stokes drag of the probe is $\zeta_{P}$, and the excluded volume fraction of the suspension is $\phi^{e x}=2 \pi a(R+a)^{2} n^{\infty} / 3$, where $n^{\infty}$ is the number density, $a$ is the swimmer size, and $R$ is the probe size. This scaled drag contribution is plotted as a function of $\ell / R_{c} \equiv U_{0} \tau_{R} /(R+a)$, where $U_{0}$ is the speed of the swimmers, and $\tau_{R}$ is their reorientation time. Different colors indicate different strengths of swimming: $6 D^{\text {swim }} / D^{\text {rel }}=U_{0}^{2} \tau_{R} / D^{\text {rel }}$, where $D^{\text {rel }}$ is the relative thermal diffusivity. Squares are for the closure $Q^{\prime}=0$ and crosses are for the closure $\boldsymbol{B}^{\prime}=\mathbf{0}$. The points are calculated from numerical solutions to the governing Eqs. (16)-(18). The dashed and solid lines serve as guides for the eye.

The effect of the bath particles is characterized by the microviscosity coefficient $\eta^{\text {micro }} \equiv\left(\langle\zeta\rangle-\zeta_{P}\right) / \zeta_{P} \phi^{e x}$, where $\zeta_{P}=6 \pi \eta_{s} R$ is the probe's Stokes drag coefficient. The microviscosity coefficient is a scalar constant independent of the volume fraction. We shall discuss the average drag in terms of the microviscosity coefficient.

\section{A. Swim-thinning}

Dimensional analysis of the governing equations for $\hat{\boldsymbol{u}} \cdot \hat{\boldsymbol{d}}^{(F)}$ reveals two dimensionless groups that determine the strength of activity in the linear-response regime: $P e_{s}=U_{0} R_{c} / D^{r e l}$ and $\gamma^{2}=2 R_{c}^{2} /\left(D^{r e l} \tau_{R}\right)$. The first is a Péclet number relating the strength of swimming to the strength of Brownian motion, and the second compares the rate of thermal diffusion to the reorientation time of the swimmers. We can define these groups in terms of three length scales: the run length $\ell=U_{0} \tau_{R}$, the microscopic length $\delta=\sqrt{D^{r e l} \tau_{R}}$, and the contact length $R_{c}\left(P e_{s}=\ell R_{c} / \delta^{2}\right.$ and $\left.\gamma^{2}=2 R_{c}^{2} / \delta^{2}\right)$. Figure 3 shows that the qualitative dependence of $\eta^{\text {micro }}$ on the ratio $\ell / R_{c}$ is the same for all activity levels-constant values of $\ell / \delta=\sqrt{6 D^{\text {swim }} / D^{r e l}} \sim \sqrt{k_{s} T_{s} / k_{B} T}$.

The limit $\ell / R_{c} \ll 1$ is known as the continuum limit. The run-length of the swimmers is small compared to the size of the probe; thus, from the probe's perspective, the swimmers are simply "hot" Brownian particles diffusing through the fluid with an active self-diffusivity $D_{T}+D^{\text {swim }}$. When they collide with the probe due to the advective flux (see Fig. 4, left), they can immediately reorient and diffuse away from the probe. Thus, even though the number density of particles at the surface may be large [see (C6)] $n_{0} \sim n^{\infty}\left(1+D^{\text {swim }} / D_{T}\right)$, the advective disturbance is balanced by the diffusive restoring flux $\left(D_{T}+D^{\text {swim }}\right) \nabla n^{\prime}$. The microviscosity coefficient is

$$
\eta^{\text {micro }}=1-\frac{\sqrt{3}}{2} \frac{\ell}{R_{c}},
$$

which is what we find in passive suspensions, minus a small correction that reflects swim-thinning of the suspension.

In the opposite limit, the run length of the swimmers is far larger than the contact length $\ell \gg R_{c}$. When a swimmer collides with the probe, it slides along the contact surface until it is able to swim away again with the same orientation (see Fig. 4, right). This is a consequence of the no-flux boundary condition. The swimmer does not reorient during its contact with the probe, and thus, the particles do not accumulate at the surface of the probe $n_{0} \sim n^{\infty}$ [see (C6)].
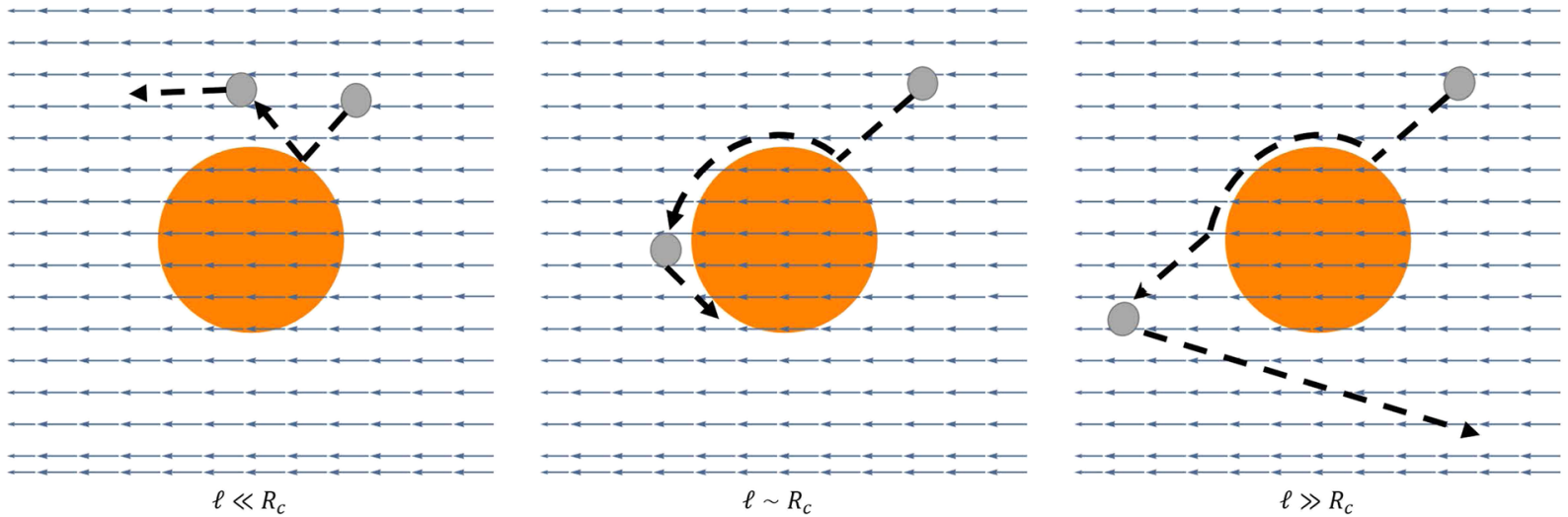

FIG. 4. Sketch of swimmer trajectories upon collision with the probe particle for various regimes of $\ell / R_{c}=U_{0} \tau_{R} /(R+a)$, where $U_{0}$ is the speed of the swimmer, $\tau_{R}$ is its reorientation time, $a$ is its size, and $R$ is the size of the probe. The background arrows indicate the direction of fluid flow. 
In this case, the weak advective perturbation is simply perturbing a suspension from a uniform microstructure, and the microviscosity coefficient is

$$
\eta^{\text {micro }}=1-\frac{2}{9} P e_{s}^{2},
$$

which is the passive suspension microviscosity coefficient with a small correction for swim-thinning when $\ell / R_{c}$ is large but finite. Note that $P e_{s}=(\ell / \delta)^{2}\left(R_{c} / \ell\right)$ is actually small when $\ell / \delta$ is fixed but $\ell / R_{c}$ becomes large.

In the intermediate regime- $\ell / R_{c} \sim O(1)$, illustrated in the center panel of Fig. 4-the behavior is different. When a swimmer collides with the probe, it slides along the surface as it does when $\ell / R_{c} \gg 1$, but it may still be near the probe when it reorients. For example, a swimmer approaching the front of the probe may collide and then slide around to the back of the probe. Once there, it can either reorient and swim away from the probe, or it can collide with the probe again and push it along-the latter scenario would result in a microviscosity coefficient less than one would find in a passive suspension. The opposite would be true of a particle approaching the rear of the swimmer-it could swim away in front or reorient and hit the probe, thereby increasing the microviscosity coefficient. When $\ell / R_{c} \sim O(1)$, we find

$$
\eta^{\text {micro }}=\sqrt{8} / P e_{s}
$$

which approaches zero as $P e_{s} \rightarrow \infty$. This simply means that the weak advective disturbance causes more swimmers to collide with the front of the probe and then slide to the back than vice versa. Some of these swimmers reorient and push the probe along, decreasing the microviscosity coefficient.

It should be noted that this particular limit of the microviscosity coefficient $-\ell / R_{c} \sim O(1)$-is sensitive to the equation closure, as indicated by the difference between square symbols and crosses in Fig. 3 (for a detailed discussion, see Appendix D). If nematic order is included, $\eta^{\text {micro }}$ approaches a constant value slightly less than $1 / 2$, as $P e_{s} \rightarrow \infty$, which is consistent with finite element calculations of $\hat{P}_{1 / 1}$ that do not rely on the moment expansion in $\boldsymbol{q}$. Additionally, the overshoot in the approach to the large $\ell / R_{c}$ value seen in Fig. 3 at large $D^{\text {swim }} / D^{\text {rel }}$ is a result of the closure $\boldsymbol{B}^{\prime}=0$. It is not seen with the closure $\boldsymbol{Q}^{\prime}=0$ or in finite-element calculations (Appendix D).

In the absence of any hydrodynamic interactions, activity decreases the excess drag from the bath particles, but the microviscosity is never reduced below the viscosity of the embedding solvent, $\langle\eta\rangle>\eta_{s}$. This agrees with previous simulations which compute a decreased mobility in an active bath without hydrodynamic interactions. $^{30}$ However, Reichhardt and Reichhardt ${ }^{30}$ found that the mobility monotonically decreases-the drag increases-with $\ell / R_{c}$ at fixed $\mathrm{Pe}_{s}$, the opposite of what we find here. It should be noted that their simulations have no Brownian motion and, thus, cannot be in the linear response regime. Their findings of an effective drag coefficient greater than the Stokes drag stand in contrast to many theoretical predictions and measurements of reduced shear viscosity in bacterial suspensions ${ }^{21,22,24,44}$ and reduced microviscosity in an active nematic.

\section{B. Connections to a generalized SES relation}

To connect dissipation to fluctuation, in addition to the probe drag, we need its diffusivity. In Fig. 5, we reframe the results of

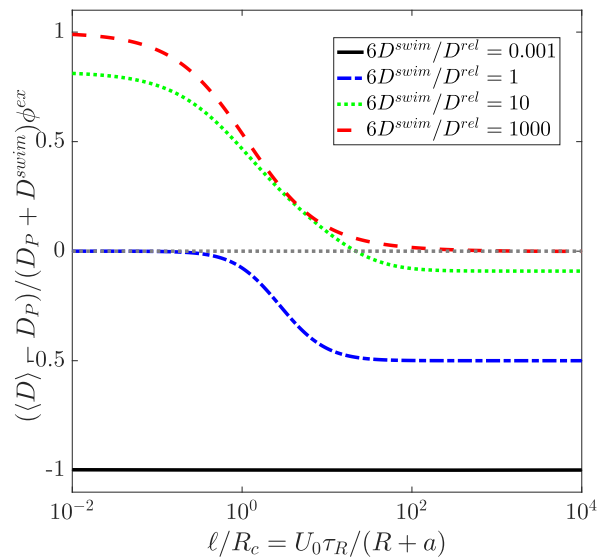

FIG. 5. Particle contribution to the probe diffusivity scaled by $\left(D_{P}+D^{\text {swim }}\right) \phi^{e x}$. The SES diffusivity of the probe is $D_{P}$, and the excluded volume fraction of the suspension is $\phi^{e x}=2 \pi a(R+a)^{2} n^{\infty} / 3$, where $n^{\infty}$ is the number density, $a$ is the swimmer size, and $R$ is the probe size. $D^{\text {swim }}=U_{0}^{2} \tau_{R} / 6$ is the swim diffusivity, where $U_{0}$ is the constant speed of the swimmers and $\tau_{R}$ is the reorientation time of a swimmers. This is plotted as a function of $\ell / R_{c} \equiv U_{0} \tau_{R} /(R+a)$. Different colors indicate different strengths of swimming, $6 D^{\text {swim }} / D^{r e l}=U_{0}^{2} \tau_{R} / D^{r e l}$, where $D^{\text {rel }}$ is the relative thermal diffusivity.

Burkholder and Brady ${ }^{28}$ for the diffusivity of a probe particle of radius $R$ in a dilute bath of active particles in the context of the present discussion.

As mentioned previously, when $\ell / R_{c} \ll 1$, the probe sees the swimmers as "hot" Brownian particles with effective diffusivity $D_{T}+D^{\text {swim }}$. This results in an enhanced probe diffusivity that scales as $k_{s} T_{s}$, as seen in Fig. 5 when $D^{\text {swim }} \gg D_{T}\left(k_{s} T_{s} \gg k_{B} T\right)$. This enhanced diffusivity is in competition with the usual steric hindrance $-D_{P} \phi^{e x}$, and when $k_{s} T_{s} / k_{B} T \ll 1$, the steric hindrance dominates (the black line in Fig. 5). In the opposite limit, $\ell / R_{c} \gg 1$ (the right-hand side of Fig. 5), the active contribution to the probe's diffusivity decreases ${ }^{28}$ because the swimmers can no longer share their full activity with the probe. When an active particle collides with the probe it can only displace it of order the probe size $R$, not its run length $\ell$, and thus, the diffusivity when scaled with $D^{\text {swim }}$ decreases with $\ell / R_{c}$. The plateaus are of differing heights due to the factor of $D^{\text {swim }}$ in the denominator; the active enhancement to the diffusivity can be smaller than the steric hindrance in this limit resulting in the negative values in Fig. 5.

In a dilute suspension of passive Brownian hard spheres in the absence of $\mathrm{HI}$, Squires and Brady showed that $\langle\zeta\rangle=1 /\langle\mu\rangle=\zeta_{P} /\left(1-\phi^{e x}\right)$, while Zia and Brady later showed $\langle D\rangle=D_{P}\left(1-\phi^{e x}\right)$ using generalized Taylor dispersion theory. ${ }^{32,43}$ The product of $\langle D\rangle$ and $1 /\langle\mu\rangle$ is, thus, precisely $k_{B} T$; as we show here, the FDT holds for all concentrations and conditions for an equilibrium suspension.

For active systems, we can investigate the validity of the FDT in the following manner. To leading order in the concentration of bath particles, the diffusivity and mobility can be written as $\langle D\rangle=D_{P}+\alpha \phi^{e x}$ and $\langle\mu\rangle=\mu_{P}+\beta \phi^{e x}$, respectively, where $\mu_{P}=1 / \zeta_{P}$ is the probe's Stokes mobility. The drag is thus $\langle\zeta\rangle=1 /\langle\mu\rangle=\zeta_{P} /\left(1+\zeta_{P} \beta \phi^{e x}\right)$. The functions $\alpha$ and $\beta$ are contained in 
Figs. 3 and 5. The product of the diffusivity and drag is thus

$$
\langle D\rangle\langle\zeta\rangle=\frac{\zeta_{P} D_{P}+\zeta_{P} \alpha \phi^{e x}}{1+\zeta_{P} \beta \phi^{e x}} \sim k_{B} T+\zeta_{P} \phi^{e x}\left(\alpha-k_{B} T \beta\right), \phi^{e x} \ll 1 .
$$

For passive particles, $\alpha-k_{B} T \beta=0$, recovering the FDT. We can thus characterize the departure from the FDT due to nonequilibrium effects-in this case, the activity of the bath particles-by examining how $\alpha-k_{B} T \beta$ varies with activity. This quantity can be thought of as an "excess" energy $E^{a c t}$ imparted to the probe by the active bath

$$
E^{a c t} \equiv \zeta_{P}\left(\alpha-k_{B} T \beta\right) \phi^{e x} .
$$

In Fig. 6, we plot these nonequilibrium fluctuations, $E^{a c t} /\left(k_{s} T_{s} \phi^{\text {ex }}\right)$, of the probe particle as a function of the quantity $\ell / R_{c}$, similar to the plots of the self-drag and self-diffusivity above. In the continuum limit, $\ell / R_{c} \ll 1$, the excess fluctuations of the probe are $k_{s} T_{s} \phi^{e x}$, independent of how active the swimmers are. Here, the run length is small compared to the contact length $\ell / R_{c} \ll 1$, so the probe sees the swimmers as effectively Brownian particles with an active self-diffusivity $D^{a c t}=D_{T}+D^{\text {swim }}$. The probe experiences many small kicks of $O\left(k_{B} T\right)$ from the solvent molecules, and kicks of $O\left(k_{s} T_{s}\right)$ from the swimmers. The volume fraction dependence of $E^{\text {act }}$ necessarily reflects the fact that the active fluctuations come from the particles only, whereas thermal fluctuations come from the solvent.

For fixed levels of activity, Fig. 6 shows that as the persistence length of the swimmers' increases, these nonequilibrium fluctuations go to zero. In this limit, the active contribution to the probe's diffusivity also vanishes ${ }^{28}$ because the swimmers can no longer share their full activity with the probe particle. In addition, recall from the discussion of the microviscosity coefficient that the microstructure of the suspension is essentially uniform in the absence of probe motion when $\ell \gg R_{c}$. The swimmers simply keep moving along their

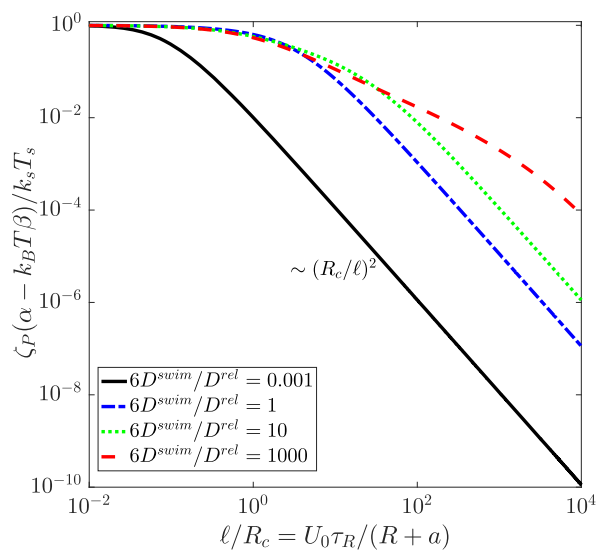

FIG. 6. Particle contribution to the probe fluctuations scaled by $k_{s} T_{s}$. The activity of the swimmers is $k_{s} T_{s}=\zeta_{s} U_{0}^{2} \tau_{R} / 6$, where $U_{0}$ is the constant speed of the swimmers and $\tau_{R}$ is the reorientation time of a swimmer, and $\zeta_{S}$ is their Stokes drag. This is plotted as a function of $\ell / R_{c} \equiv U_{0} \tau_{R} /(R+a)$. Different colors indicate different strengths of swimming: $6 D^{s w i m} / D^{r e l}=U_{0}^{2} \tau_{R} / D^{r e l} \sim k_{s} T_{s} / k_{B} T$, where $D^{r e l}$ is the relative thermal diffusivity. desired trajectories upon collisions with the probe. Thus, the diffusive motion of the probe is not affected by the activity of the bath, and the FDT holds true.

That the FDT applies in the limit of large $\ell / R_{c}$ is really a statement that activity no longer matters. As defined in (29) the when the fluctuation is zero $\langle D\rangle\langle\zeta\rangle=k_{B} T$. The effect of activity on the probe can only come about through "collisions" with the active bath particles and thus is fundamentally proportional to their volume fraction $\phi^{e x}$. Thus, the FDT that we should expect is one where $\langle D\rangle\langle\zeta\rangle=k_{B} T+k_{s} T_{s} \phi^{e x}$, where the coefficient of $\phi^{e x}$ is only the activity $k_{s} T_{s}$, which is what we see in the limit of small $\ell / R_{c}$.

This motivates us to look more carefully at the excess energy $E^{a c t}$ imparted to the probe by the active bath. One can employ kinematic arguments analogous to those used in Burkholder and Brady $^{28}$ to examine the functional forms of the excess energy to elicit the dependence on particle activity and suspension geometry/microstructure. Rather than to confuse the reader with the introduction of time scales used in that paper, we can examine the limiting behavior in terms of $\ell / R_{c}$.

(1) In the continuum limit $\ell \ll R_{c}$, the probe is in a "hot" bath of Brownian particles, where the total temperature of the bath is $k_{B} T+k_{s} T_{s} \phi^{e x}$. Accordingly, the nonequilibrium fluctuations of the probe are

$$
E^{a c t}=k_{s} T_{s}\left(\frac{R}{R_{c}}\right) \phi^{e x},
$$

just as in the simple result for a single ABP. In the limit $a / R \rightarrow 0, E^{a c t}=k_{s} T_{s} \phi^{e x}$, and in the limit $a / R \rightarrow \infty, E^{a c t}=k_{s} T_{s} \phi$.

(2) When the swimmers are persistent $\left(\ell \gg R_{c}\right)$, the detailed calculations give

$$
E^{a c t}=\frac{34}{9} k_{s} T_{s}\left(\frac{k_{s} T_{s}}{k_{B} T}\right)\left(\frac{R}{\ell}\right)^{2} \phi^{e x},
$$

regardless of the swimmer-to-probe size ratio $a / R$ (when $a / R \ll 1$ the bare volume fraction $\phi$ takes the place of the excluded volume fraction $\left.\phi^{e x}\right)$. This is consistent with the scaled nonequilibrium energy decaying to zero as $(R / \ell)^{2}$ in Fig. 6.

(3) In the intermediate regime, where $\ell \sim R_{c}$, we find

$$
E^{a c t}=\frac{2}{\sqrt{3}} k_{s} T_{s}\left(\frac{R}{\ell}\right)\left(\frac{2+\sqrt{2} R_{c} / \delta}{1+\sqrt{2} R_{c} / \delta}\right) \phi^{e x} .
$$

In this limit, a swimmer collides with the probe and remains nearby when it reorients. This result is also insensitive to the swimmer-probe size ratio $a / R$. It manifests only in $\phi^{e x}$, which simply becomes $\phi$ for point tracers.

In general then, the fluctuation-dissipation relation in active systems reads

$$
\langle\boldsymbol{D}\rangle \cdot\left\langle\boldsymbol{M}^{U F}\right\rangle^{-1}=k_{B} T \boldsymbol{I}+\boldsymbol{E}^{a c t}\left(a / R, \phi, \ell / R_{c}, \ldots\right),
$$

which is a weaker statement than the FDT for equilibrium systems. Indeed, though $\boldsymbol{E}^{a c t}$ is isotropic in the case studied in this article, it may be anisotropic in other scenarios. For the cases studied here $E^{a c t} \sim k_{s} T_{s} \phi^{e x}$, which is linear in the activity and concentration of active bath particles, but the coefficient that may depend on $\ell / R_{c}$, etc. Only in the continuum limit $\ell / R_{c} \rightarrow 0$ do we find that the product 
$\langle D\rangle \cdot\left\langle M^{U F}\right\rangle^{-1}$ depends solely activity of the bath particles $k_{s} T_{s} \phi^{e x}-$ in this limit the probe is large enough that the random active motion of the bath particles is instantaneous and microscopic on the scale of its own motion, just like in an equilibrium suspension. Finally, though these calculations specifically address 3-D systems, the same physical arguments are expected to apply in 2-D.

\section{CONCLUSIONS}

Using generalized Taylor dispersion theory, we showed that, although the long-time self-diffusivity and self-mobility are formally identical the displacement fields are not, and the fluctuationdissipation theorem (FDT) does not hold in general for nonequilibrium systems. Nevertheless, for a dilute active bath we showed that a modified form of the FDT applies with the product of the diffusivity and drag now proportional to the thermal energy, $k_{B} T$ plus the active energy, $E^{a c t} \sim k_{s} T_{s} \phi^{e x}$, the latter of which may also depend on the ratio of the run length to probe size, $\ell / R_{c}$, etc. In the continuum limit where the run length of the swimmer is much smaller than the center-to-center separation of the probe and swimmer upon contact $\ell \ll R_{c}$ the active energy is strictly promotional to $k_{s} T_{s}$ and the modified FDT is valid.

In finding the effective fluctuation-dissipation relation, we necessarily calculated the linear viscous response of an active suspension to a tracer particle being dragged by a fixed external force. We find that the suspension, in the absence of hydrodynamic interactions (HI), is uniformly swim-thinning. The microviscosity coefficient reaches a minimum value of approximately $1 / 2$ of that seen in passive suspensions when the run length and contact length are commensurate $\ell \sim R_{c}$; this is illustrated in the center of Fig. 3. The precise value of the minimum predicted microviscosity coefficient will depend on the closure of the Smoluchowski equation.

Notably, we did not find a negative microviscosity in this investigation-the swimmers always increase the effective suspension microviscosity no matter how active they become, as demonstrated in simulations ${ }^{30}$ but in contrast to measurements of the microviscosity of an active nematic. ${ }^{23}$ There are two explanations for this. First, we have implicitly assumed a fixed-force mode of microrheology in using generalized Taylor dispersion theory. Negative suspension viscosities are precluded in this mode of measurement because a negative viscosity would result in the speed of the probe increasing until the effective drag was once again positive. ${ }^{44}$ No matter how small the pulling force, as the viscosity goes to zero the probe speed increases to where the relevant Péclet number for the motion is no longer small.

Second, in neglecting HI we do not consider any effects related to the swimmers' gait, as in previous theoretical investigations. ${ }^{22,23}$ We expect that hydrodynamic interactions are essential to finding the observed viscosity reduction in active suspensions. Even in the case where the swimmers exert no hydrodynamic stress on the fluid, Takatori and Brady ${ }^{44}$ showed that the swim stress-the macroscopic mechanical stress associated with the random runand-tumble motion of the active particles-is affected by an ambient viscometric flow, and indeed they predict a viscosity reduction in active suspensions regardless of swimming gait or shape of the swimmers. We examine the effects of $\mathrm{HI}$ in a forthcoming study.

\section{ACKNOWLEDGMENTS}

This work was supported in part by the NSF under Grant No. CBET 1437570.

\section{APPENDIX A: GENERALIZED TAYLOR DISPERSION THEORY IN NONEQUILIBRIUM SYSTEMS}

Consider a suspension of $N$ particles embedded in a Newtonian fluid. The suspension dynamics may be described by a Smoluchowski equation for $P_{N}\left(\left\{\boldsymbol{x}_{\alpha}\right\}, t\right)$, the probability of finding the $N$ particles in some phase-space configuration $\left\{\boldsymbol{x}_{\alpha}\right\}$ at time $t\left(\boldsymbol{x}_{\alpha}\right.$ is taken to represent the laboratory frame position and orientation of each particle)

$$
\frac{\partial P_{N}}{\partial t}+\sum_{\alpha=1}^{N} \nabla_{\alpha} \cdot \boldsymbol{j}_{\boldsymbol{\alpha}}=0
$$

with the general constitutive expression for the flux $\boldsymbol{j}_{\alpha}$ :

$$
\boldsymbol{j}_{\boldsymbol{\alpha}}=\boldsymbol{U}_{\alpha} P_{N}-\sum_{\beta=1}^{N} \boldsymbol{D}_{\alpha \beta} \cdot \nabla_{\beta} P_{N} .
$$

The thermal diffusivity of particle $\alpha$ relative to $\beta$ is described by $\boldsymbol{D}_{\alpha \beta}=k_{B} T \boldsymbol{M}_{\boldsymbol{\alpha} \beta}^{U \boldsymbol{U}}$, where $\boldsymbol{M}_{\boldsymbol{\alpha} \boldsymbol{U}}^{\boldsymbol{U F}}$ is the hydrodynamic mobility ${ }^{31,45}$ coupling the velocity of particle $\alpha, \boldsymbol{U}_{\boldsymbol{\alpha}}$ to the force on particle $\beta, \boldsymbol{F}_{\beta}$. We take $\boldsymbol{U}$ to represent both translational and angular velocities of the particles, and $\boldsymbol{F}$ to represent both forces and torques. We have explicitly written the particle fluxes arising from Brownian motion; the velocity $\boldsymbol{U}_{\boldsymbol{\alpha}}$ contains all other contributions to the particle motion, whether it be from activity, external forces or flows, or interparticle forces.

To probe the suspension properties, we select a test particle at $\boldsymbol{x}_{1} \equiv \boldsymbol{z}$, and rewrite the equation in terms of particle coordinates relative to the test particle $\boldsymbol{r}_{\alpha}=\boldsymbol{x}_{\alpha}-\boldsymbol{x}_{1}$

$$
\frac{\partial P_{N}}{\partial t}+\nabla_{z} \cdot \boldsymbol{j}_{\mathbf{1}}+\sum_{\alpha=1}^{N} \nabla_{r_{\alpha}} \cdot\left(\boldsymbol{j}_{\boldsymbol{\alpha}}-\mathbf{j}_{\mathbf{1}}\right)=0,
$$

where

$$
\begin{aligned}
& \boldsymbol{j}_{\mathbf{1}}=\boldsymbol{U}_{\mathbf{1}} P_{N}-\boldsymbol{D}_{\mathbf{1 1}} \cdot \nabla_{z} P_{N}-\sum_{\alpha=1}^{N}\left(\boldsymbol{D}_{\mathbf{1} \boldsymbol{\alpha}}-\boldsymbol{D}_{\mathbf{1 1}}\right) \cdot \nabla_{r_{\alpha}} P_{N}, \\
& \boldsymbol{j}_{\boldsymbol{\alpha}}=\boldsymbol{U}_{\boldsymbol{\alpha}} P_{N}-\boldsymbol{D}_{\boldsymbol{\alpha} \mathbf{1}} \cdot \nabla_{z} P_{N}-\sum_{\beta=1}^{N}\left(\boldsymbol{D}_{\boldsymbol{\alpha} \boldsymbol{\beta}}-\boldsymbol{D}_{\boldsymbol{\alpha} \mathbf{1}}\right) \cdot \nabla_{r_{\beta}} P_{N},
\end{aligned}
$$

according to the chain rule. ${ }^{46}$

Fluctuations in the test-particle coordinate $z$ are unbounded, so we may take a Fourier transform with respect to $z$ derive

$$
\frac{\partial \hat{P}_{N}}{\partial t}+i \boldsymbol{k} \cdot \hat{\boldsymbol{j}}_{\mathbf{1}}+\sum_{\alpha=1}^{N} \nabla_{r_{\alpha}} \cdot\left(\hat{\boldsymbol{j}}_{\boldsymbol{\alpha}}-\hat{\boldsymbol{j}}_{\mathbf{1}}\right)=0,
$$

where

$$
\begin{aligned}
& \hat{\boldsymbol{j}}_{\mathbf{1}}=\boldsymbol{U}_{\mathbf{1}} \hat{P}_{N}-i \boldsymbol{k} \cdot \boldsymbol{D}_{\mathbf{1 1}} \hat{P}_{N}-\sum_{\alpha=1}^{N}\left(\boldsymbol{D}_{\mathbf{1} \boldsymbol{\alpha}}-\boldsymbol{D}_{\mathbf{1 1}}\right) \cdot \nabla_{r_{\alpha}} \hat{P}_{N} \\
& \hat{\boldsymbol{j}}_{\boldsymbol{\alpha}}=\boldsymbol{U}_{\boldsymbol{\alpha}} \hat{P}_{N}-i \boldsymbol{k} \cdot \boldsymbol{D}_{\boldsymbol{\alpha} \mathbf{1}} \hat{P}_{N}-\sum_{\beta=1}^{N}\left(\boldsymbol{D}_{\boldsymbol{\alpha} \beta}-\boldsymbol{D}_{\boldsymbol{\alpha} \mathbf{1}}\right) \cdot \nabla_{r_{\beta}} \hat{P}_{N} .
\end{aligned}
$$


We have assumed that the velocity of the test particle is independent of its absolute position $z$. This may not be true if, for example, the particle experiences motion due to an external potential fixed in the laboratory frame.

We may average the $N$-particle equation over the relative configurations of the $N-1$ other particles to get an equation of motion for the test particle

$$
\frac{\partial \hat{P}_{1}}{\partial t}+i \boldsymbol{k} \cdot\left\langle\hat{\boldsymbol{j}}_{\mathbf{1}}\right\rangle_{N-1}=0
$$

which makes use of the condition that there be no relative particle flux at contact or at infinity: $\boldsymbol{n}_{\mathbf{1} \boldsymbol{\alpha}} \cdot\left(\hat{\boldsymbol{j}} \boldsymbol{\alpha}_{\boldsymbol{\alpha}}-\hat{\boldsymbol{j}}_{\mathbf{1}}\right)=0$, where $\boldsymbol{n}_{\mathbf{1} \boldsymbol{\alpha}}$ is the unit vector pointing from particle 1 to particle $\alpha$ along the line of center. The average flux of the test particle is

$$
\begin{aligned}
\frac{\left\langle\hat{\boldsymbol{j}}_{\mathbf{1}}\right\rangle_{N-1}}{\hat{P}_{1}}= & \int \boldsymbol{U}_{\mathbf{1}} \hat{P}_{N-1 / 1} d \boldsymbol{r}_{\boldsymbol{\alpha}}-i \boldsymbol{k} \cdot \int \boldsymbol{D}_{\mathbf{1 1}} \hat{P}_{N-1 / 1} d \boldsymbol{r}_{\boldsymbol{\alpha}} \\
& -\int \sum_{\alpha=1}^{N}\left(\boldsymbol{D}_{\mathbf{1} \boldsymbol{\alpha}}-\boldsymbol{D}_{\mathbf{1 1}}\right) \cdot \nabla_{r_{\alpha}} \hat{P}_{N-1 / 1} d \boldsymbol{r}_{\boldsymbol{\alpha}},
\end{aligned}
$$

which has been simplified by the substitution

$$
\hat{P}_{N}=\hat{P}_{1}(\boldsymbol{k}, t) \hat{P}_{N-1 / 1}\left(\boldsymbol{k},\left\{\boldsymbol{r}_{\alpha}\right\}, t\right) .
$$

The long-time self-diffusivity of the test particle will be determined by the long wave length (small $\boldsymbol{k}$ ) fluctuations in its position so we expand the relative microstructure with respect to $i \boldsymbol{k}: \hat{P}_{N-1 / 1}=\hat{P}_{N-1 / 1}^{(0)}+i \boldsymbol{k} \cdot \hat{\boldsymbol{d}}^{(\boldsymbol{k})}+O\left(k^{2}\right)$. From this, we can define an effective dispersion relation

$$
\left\langle\hat{\boldsymbol{j}}_{1}\right\rangle_{N-1} \equiv\left[\left\langle\boldsymbol{U}^{\text {probe }}\right\rangle-i \boldsymbol{k} \cdot\langle\boldsymbol{D}\rangle+\ldots\right] \hat{P}_{1},
$$

where

$$
\left\langle\boldsymbol{U}^{\text {probe }}\right\rangle=\int \boldsymbol{U}_{\mathbf{1}} \hat{P}_{N-1 / 1}^{(0)} d \boldsymbol{r}_{\boldsymbol{\alpha}}-\int \sum_{\alpha=1}^{N}\left(\boldsymbol{D}_{\mathbf{1 \alpha}}-\boldsymbol{D}_{\mathbf{1 1}}\right) \cdot \nabla_{r_{\alpha}} \hat{P}_{N-1 / 1}^{(0)} d \boldsymbol{r}_{\boldsymbol{\alpha}},
$$

$$
\begin{aligned}
\langle\boldsymbol{D}\rangle= & \int \boldsymbol{D}_{\mathbf{1 1}} \hat{P}_{N-1 / 1}^{(0)} d \boldsymbol{r}_{\boldsymbol{\alpha}}-\int \boldsymbol{U}_{\mathbf{1}} \hat{\boldsymbol{d}}^{(\boldsymbol{k})} d \boldsymbol{r}_{\boldsymbol{\alpha}} \\
& +\int \sum_{\alpha=1}^{N}\left(\boldsymbol{D}_{\mathbf{1} \boldsymbol{\alpha}}-\boldsymbol{D}_{\mathbf{1 1}}\right) \cdot \nabla_{r_{\alpha}} \hat{\boldsymbol{d}}^{(\boldsymbol{k})} d \boldsymbol{r}_{\boldsymbol{\alpha}} .
\end{aligned}
$$

This is a generalization of the 2-particle result for equilibrium suspensions derived in Ref. 32; recall that $\boldsymbol{U}_{1}$ can have nonequilibrium contributions, which contribute to the diffusivity through $\hat{\boldsymbol{d}}^{(k)}$. Note that the last integral in Eq. (A13) is convergent for a quadrupolar fluctuation field, and conditionally convergent for a dipolar fluctuation. We return to this point later.

Consider separately the motion of the test particle due to an applied external force, $U_{1}=U_{1}^{(0)}+M_{11}^{U F} \cdot F^{\text {ext }}$, where $U_{1}^{(0)}$ can still contain contributions from shear flow, activity, etc. The average velocity of the test particle is now

$$
\begin{aligned}
\left\langle\boldsymbol{U}^{\text {probe }}\right\rangle= & \int\left(\boldsymbol{U}_{\mathbf{1}}^{(\mathbf{0})}+\boldsymbol{M}_{\mathbf{1 1}}^{U F} \cdot \boldsymbol{F}^{\text {ext }}\right) \hat{P}_{N-1 / 1}^{(0)} d \boldsymbol{r}_{\boldsymbol{\alpha}} \\
& -\int \sum_{\alpha=1}^{N}\left(\boldsymbol{D}_{\mathbf{1} \boldsymbol{\alpha}}-\boldsymbol{D}_{\mathbf{1 1}}\right) \cdot \nabla_{r_{\alpha}} \hat{P}_{N-1 / 1}^{(0)} d \boldsymbol{r}_{\boldsymbol{\alpha}} .
\end{aligned}
$$

If the external force is weak, we can also do a regular perturbation expansion of the microstructure in moments of the force: $\hat{P}_{N-1 / 1}^{(0)}=\hat{P}_{N-1 / 1}^{(0,0)}-\boldsymbol{F}^{\text {ext }} \cdot \hat{\boldsymbol{d}}^{(F)}+O\left(F^{\text {ext }}\right)^{2}$. The fluctuation field arising from the external force has units of length per energy; one could also define the wave vector $\boldsymbol{F}^{\text {ext }} / k_{B} T$ instead so that the units of $\hat{\boldsymbol{d}}^{(F)}$ are also length. The average test particle velocity is

$$
\begin{aligned}
\left\langle\boldsymbol{U}^{\text {probe }}\right\rangle= & \left(\int \boldsymbol{U}_{\mathbf{1}}^{(\mathbf{0})} \hat{P}_{N-1 / 1}^{(0,0)} d \boldsymbol{r}_{\alpha}-\int \sum_{\alpha=1}^{N}\left(\boldsymbol{D}_{\mathbf{1} \boldsymbol{\alpha}}-\boldsymbol{D}_{\mathbf{1 1}}\right) \cdot \nabla_{r_{\alpha}} \hat{P}_{N-1 / 1}^{(0,0)} d \boldsymbol{r}_{\boldsymbol{\alpha}}\right) \\
& +\boldsymbol{F}^{\boldsymbol{e x t}} \cdot\left(\int \boldsymbol{M}_{\mathbf{1 1}}^{U F} \hat{P}_{N-1 / 1}^{(0,0)} d \boldsymbol{r}_{\boldsymbol{\alpha}}-\int \boldsymbol{U}_{\mathbf{1}}^{(\mathbf{0})} \hat{\boldsymbol{d}}^{(\boldsymbol{F})} d \boldsymbol{r}_{\boldsymbol{\alpha}}\right. \\
& \left.+\int \sum_{\alpha=1}^{N}\left(\boldsymbol{D}_{\mathbf{1} \boldsymbol{\alpha}}-\boldsymbol{D}_{\mathbf{1 1}}\right) \cdot \nabla_{r_{\alpha}} \hat{\boldsymbol{d}}^{(F)} d \boldsymbol{r}_{\boldsymbol{\alpha}}\right) .
\end{aligned}
$$

If we define $\left\langle\boldsymbol{U}^{\text {probe }}\right\rangle \equiv\left\langle\boldsymbol{U}^{\text {drift }}\right\rangle+\left\langle\boldsymbol{M}^{U F}\right\rangle \cdot \boldsymbol{F}^{\text {ext }}$, then we have an expression for $\left\langle\boldsymbol{U}^{\text {drift }}\right\rangle$ that is formally identical to Eq. (A12), and an expression for $\left\langle\boldsymbol{M}^{\boldsymbol{U F}}\right\rangle$ that is formally identical to Eq. (A13). From this generalized Taylor dispersion perspective, dispersion driven by Brownian motion of the bath particles is equivalent to dispersion driven by an applied external force $\boldsymbol{F}^{\boldsymbol{e x t}}=-i \boldsymbol{k} \boldsymbol{k}_{B} T$.

\section{APPENDIX B: GOVERNING EQUATIONS FOR THE FLUCTUATION FIELDS}

The fluctuation-dissipation theorem for colloidal suspensions requires that the long-time self-diffusivity is proportional to the hydrodynamic mobility $\langle\boldsymbol{D}\rangle=k_{B} T\left\langle\boldsymbol{M}^{U F}\right\rangle$-a result derived by Einstein, Sutherland, and von Smoluchowski independently. Qualitatively, this states that the random fluctuating motion of a test particle is related to the effective drag that the test particle would feel if it were pulled through the fluid by an external force. Although the expressions for the mobility and diffusivity are formally identical (modulo a factor of $k_{B} T$ ), the fluctuation-dissipation theorem is satisfied only if $\hat{\boldsymbol{d}}^{(\boldsymbol{k})}=k_{B} T \hat{\boldsymbol{d}}^{(F)}$. We can derive equations for the fluctuation fields from Eq. (A6) from substitution of Eq. (A9) and $\hat{P}_{N}=\hat{P}_{1}(\boldsymbol{k}, t) \hat{P}_{N-1 / 1}\left(\boldsymbol{k},\left\{\boldsymbol{r}_{\alpha}\right\}, t\right)$

$\frac{\partial \hat{P}_{N-1 / 1}}{\partial t}+\sum_{\alpha=1}^{N} \nabla r_{\alpha} \cdot\left(\hat{\boldsymbol{j}}_{\boldsymbol{\alpha}}-\hat{\boldsymbol{j}}_{\mathbf{1}}\right)=i \boldsymbol{k} \cdot\left(\left[\left\langle\hat{\boldsymbol{j}}_{\mathbf{1}}\right\rangle_{N-1} / \hat{P}_{1}\right] \hat{P}_{N-1 / 1}-\hat{\boldsymbol{j}}_{\mathbf{1}}\right)$,

with boundary conditions of no relative flux at contact and infinite separation

$$
n_{1 \alpha} \cdot\left(\hat{j}_{\alpha}-\hat{j}_{1}\right)=0
$$

\section{Thermal fluctuations}

The governing equations for $P_{N-1 / 1}^{(0)}$ are

$$
\begin{gathered}
\frac{\partial \hat{P}_{N-1 / 1}^{(0)}}{\partial t}+\sum_{\alpha=2}^{N} \nabla_{r_{\alpha}} \cdot\left(\left[\boldsymbol{U}_{\boldsymbol{\alpha}}-\boldsymbol{U}_{\mathbf{1}}\right] \hat{P}_{N-1 / 1}^{(0)}-\boldsymbol{D}_{\alpha \beta}^{r e l} \cdot \nabla_{r_{\beta}} \hat{P}_{N-1 / 1}^{(0)}\right)=0, \\
\sum_{\alpha=2}^{N} \boldsymbol{n}_{\mathbf{1} \boldsymbol{\alpha}} \cdot\left(\left[\boldsymbol{U}_{\boldsymbol{\alpha}}-\boldsymbol{U}_{\mathbf{1}}\right] \hat{P}_{N-1 / 1}^{(0)}-\boldsymbol{D}_{\boldsymbol{\alpha} \boldsymbol{\beta}}^{r e l} \cdot \nabla_{r_{\beta}} \hat{P}_{N-1 / 1}^{(0)}\right)=0,
\end{gathered}
$$


where $D_{\alpha \beta}^{r e l} \equiv \sum_{\beta=2}^{N}\left(D_{\alpha \beta}-D_{\beta 1}-D_{1 \alpha}+D_{11}\right)$, and the fluctuation field is governed by

$$
\begin{aligned}
\frac{\partial \hat{\boldsymbol{d}}^{(\boldsymbol{k})}}{\partial t} & +\sum_{\alpha=2}^{N} \nabla_{r_{\alpha}} \cdot\left(\left[\boldsymbol{U}_{\boldsymbol{\alpha}}-\boldsymbol{U}_{\mathbf{1}}\right] \hat{\boldsymbol{d}}^{(\boldsymbol{k})}-\boldsymbol{D}_{\boldsymbol{\alpha} \boldsymbol{\beta}}^{r e l} \cdot \nabla_{r_{\beta}} \hat{\boldsymbol{d}}^{(\boldsymbol{k})}\right) \\
= & \hat{P}_{N-1 / 1}^{(0)}\left(\left\langle\boldsymbol{U}^{\text {probe }}\right\rangle-\boldsymbol{U}_{\mathbf{1}}\right)+2 \sum_{\alpha=1}^{N}\left(\boldsymbol{D}_{\mathbf{1} \boldsymbol{\alpha}}-\boldsymbol{D}_{\mathbf{1 1}}\right) \cdot \nabla_{r_{\alpha}} \hat{P}_{N-1 / 1}^{(0)} \\
& +\hat{P}_{N-1 / 1}^{(0)} \sum_{\alpha=1}^{N} \nabla_{r_{\alpha}} \cdot\left(\boldsymbol{D}_{\mathbf{1} \boldsymbol{\alpha}}-\boldsymbol{D}_{\mathbf{1 1}}\right)
\end{aligned}
$$

with the no-flux condition

$$
\begin{gathered}
\sum_{\alpha=2}^{N} \boldsymbol{n}_{\mathbf{1 \alpha}} \cdot\left(\left[\boldsymbol{U}_{\boldsymbol{\alpha}}-U_{\mathbf{1}}\right] \hat{\boldsymbol{d}}^{(k)}-\boldsymbol{D}_{\alpha \beta}^{r e l} \cdot \nabla_{r_{\beta}} \hat{\boldsymbol{d}}^{(k)}\right) \\
=\sum_{\alpha=2}^{N} \boldsymbol{n}_{\mathbf{1 \alpha}} \cdot\left(D_{1 \alpha}-D_{11}\right) \hat{P}_{N-1 / 1}^{(0)} .
\end{gathered}
$$

If we integrate Eq. (B5) with respect to $\boldsymbol{r}_{\alpha}$, apply the no-flux boundary condition (B6) and make use of $\int \hat{P}_{N-1 / 1} d \boldsymbol{r}_{\boldsymbol{\alpha}}=1$, then we find the same equation for $\left\langle\boldsymbol{U}^{\text {probe }}\right\rangle$ as given in Eq. (A12). ${ }^{50}$

For the simplest case of pairwise interactions between hard spheres at equilibrium, $\hat{\boldsymbol{d}}^{(\boldsymbol{k})}=-(1 / 4) \boldsymbol{r} / \boldsymbol{r}^{3}$. The fluctuation field is dipolar, and thus, the integral determining the effective diffusivity is conditionally convergent. Using the divergence theorem, one can convert the integral to a surface integral at particle contact and infinity

$$
-\int D_{11} \nabla_{r} \hat{\boldsymbol{d}}^{(\boldsymbol{k})} d \boldsymbol{r}=\int_{S_{c}} D_{11} \boldsymbol{n} \hat{\boldsymbol{d}}^{(\boldsymbol{k})} d S+\int_{S_{\infty}} D_{11} \boldsymbol{n} \hat{\boldsymbol{d}}^{(\boldsymbol{k})} d S .
$$

Recognizing the singular nature of the small $\boldsymbol{k}$ expansion at large interparticle distance $r$, we assume that the integral over $S_{\infty}$ is zero, when, in fact, it should precisely cancel the contribution from the surface. This is because the dipolar solution is technically not the correct far-field form for $\hat{\boldsymbol{d}}^{(\boldsymbol{k})}$. Our small $\boldsymbol{k}$ expansion of the microstructure is regular within a distance $\boldsymbol{r}_{\alpha} \sim \boldsymbol{k}^{-1}$ from the test particle, but there is a convective-diffusive boundary-layer at large distances. ${ }^{51}$ The correct far-field solution is really a screened dipole (making the surface integral over $S_{\infty}$ unconditionally convergent). If one wanted to consider these boundary-layer effects, one could solve the boundary-layer problem for all $\boldsymbol{k}$ using matched asymptotic expansions, evaluate the average flux (A7), and then take the limit for small $\boldsymbol{k}$ (see Rallison and $\mathrm{Hinch}^{52}$ ).

\section{External fluctuations}

The equation for the unforced microstructure $\hat{P}_{N-1 / 1}^{(0,0)}$ is the same as Eq. (B3) for the forced microstructure

$$
\begin{gathered}
\frac{\partial \hat{P}_{N-1 / 1}^{(0,0)}}{\partial t}+\sum_{\alpha=2}^{N} \nabla_{r_{\alpha}} \cdot\left(\left[\boldsymbol{U}_{\boldsymbol{\alpha}}^{(0)}-\boldsymbol{U}_{\mathbf{1}}^{(0)}\right] \hat{P}_{N-1 / 1}^{(0,0)}-\boldsymbol{D}_{\alpha \beta}^{r e l} \cdot \nabla_{r_{\beta}} \hat{P}_{N-1 / 1}^{(0,0)}\right)=0 \\
\sum_{\alpha=2}^{N} \boldsymbol{n}_{\mathbf{1} \alpha} \cdot\left(\left[\boldsymbol{U}_{\boldsymbol{\alpha}}^{(0)}-\boldsymbol{U}_{\mathbf{1}}^{(\mathbf{0})}\right] \hat{P}_{N-1 / 1}^{(0,0)}-\boldsymbol{D}_{\alpha \beta}^{r e l} \cdot \nabla_{r_{\beta}} \hat{P}_{N-1 / 1}^{(0,0)}\right)=0
\end{gathered}
$$

but the fluctuation field is

$$
\begin{aligned}
\frac{\partial \hat{\boldsymbol{d}}^{(F)}}{\partial t} & +\sum_{\alpha=2}^{N} \nabla_{r_{\alpha}} \cdot\left(\left[\boldsymbol{U}_{\boldsymbol{\alpha}}^{(\mathbf{0})}-\boldsymbol{U}_{\mathbf{1}}^{(\mathbf{0 )}}\right] \hat{\boldsymbol{d}}^{(F)}-\boldsymbol{D}_{\boldsymbol{\alpha} \boldsymbol{\beta}}^{r e l} \cdot \nabla_{r_{\beta}} \hat{\boldsymbol{d}}^{(F)}\right) \\
= & \sum_{\alpha=2}^{N} \nabla_{r_{\alpha}} \cdot\left(\boldsymbol{M}_{\mathbf{1} \boldsymbol{\alpha}}^{U F}-\boldsymbol{M}_{\mathbf{1 1}}^{U F}\right) \hat{P}_{N-1 / 1}^{(0,0)},
\end{aligned}
$$

with the no-flux condition

$$
\begin{gathered}
\sum_{\alpha=2}^{N} \boldsymbol{n}_{\mathbf{1} \boldsymbol{\alpha}} \cdot\left(\left[\boldsymbol{U}_{\boldsymbol{\alpha}}-\boldsymbol{U}_{\mathbf{1}}^{(\mathbf{0})}\right] \hat{\boldsymbol{d}}^{(F)}-\boldsymbol{D}_{\boldsymbol{\alpha} \boldsymbol{\beta}}^{r e l} \cdot \nabla_{r_{\beta}} \hat{\boldsymbol{d}}^{(F)}\right) \\
=\sum_{\alpha=2}^{N} \boldsymbol{n}_{\mathbf{1} \boldsymbol{\alpha}} \cdot\left(\boldsymbol{M}_{\mathbf{1} \boldsymbol{\alpha}}^{U F}-\boldsymbol{M}_{\mathbf{1 1}}^{U F}\right) P_{N-1 / 1}^{(0,0)} .
\end{gathered}
$$

The governing equations for the two different fluctuation fields are clearly different. Notably, the governing equation for $\hat{\boldsymbol{d}}^{(F)}$ is not forced by the average velocity.

Indeed, we find the following condition for the fluctuationdissipation theorem to be satisfied $\left(\langle\boldsymbol{D}\rangle=k_{B} T\left\langle\boldsymbol{M}^{U F}\right\rangle\right)$ : the local probe velocity

$$
\boldsymbol{U}^{\text {probe }}=\boldsymbol{U}_{\mathbf{1}}-\sum_{\alpha=1}^{N}\left(\boldsymbol{D}_{\mathbf{1 \alpha}}-\boldsymbol{D}_{\mathbf{1 1}}\right) \cdot \nabla_{r_{\alpha}} \ln \hat{P}_{N-1 / 1}^{(0)}
$$

must be everywhere equal to the average probe velocity as given in Eq. (A12). This can only be true if both $\boldsymbol{U}^{\text {probe }}$ and $\hat{P}_{N-1 / 1}^{(0)}$ are spatially uniform, or if $\boldsymbol{U}^{\text {probe }}=0$. For the equilibrium, hard-sphere suspension studied in Ref. 32, the probability distribution $\hat{P}_{N-1 / 1}^{(0)}$ is uniform, and $\boldsymbol{U}^{\text {probe }}=0$; the theorem is satisfied. This would also be satisfied for the Boltzmann distribution $\hat{P}_{N-1 / 1}^{(0)}=P_{0} e^{-V\left(\boldsymbol{r}_{\alpha}\right) / k_{B} T}$

$$
\left\langle\boldsymbol{U}^{\text {probe }}\right\rangle=\boldsymbol{U}_{\mathbf{1}}+\sum_{\alpha=1}^{N}\left(\boldsymbol{M}_{1 \boldsymbol{\alpha}}^{U \boldsymbol{F}}-\boldsymbol{M}_{\mathbf{1 1}}^{U F}\right) \cdot \nabla_{r_{\alpha}} V .
$$

The second term will precisely cancel the velocity of particle one due to interparticle forces. If there are no other forces acting on the test particle (e.g., no shear), then the fluctuation dissipation relation is again satisfied.

\section{Quantifying departures from the FD theorem}

We define a difference field between force-induced and thermally induced fluctuations, $\hat{\boldsymbol{d}}^{(k)}=k_{B} T \hat{\boldsymbol{d}}^{(F)}+\boldsymbol{d}^{\prime}$, and assert that $\boldsymbol{F}^{\boldsymbol{e x t}}=-i \boldsymbol{k} \boldsymbol{k}_{B} T$. We can then subtract the equations for the thermal and external fluctuations to find

$$
\begin{aligned}
\frac{\partial \boldsymbol{d}^{\prime}}{\partial t} & +\sum_{\alpha=2}^{N} \nabla_{r_{\alpha}} \cdot\left(\left[\boldsymbol{U}_{\boldsymbol{\alpha}}-\boldsymbol{U}_{\mathbf{1}}^{(\mathbf{0})}\right] \boldsymbol{d}^{\prime}-\boldsymbol{D}_{\boldsymbol{\alpha} \boldsymbol{\beta}}^{r e l} \cdot \nabla_{r_{\beta}} \boldsymbol{d}^{\prime}\right) \\
& =\hat{P}_{N-1 / 1}^{(0,0)}\left(\boldsymbol{U}^{\text {probe }}-\boldsymbol{U}_{\mathbf{1}}\right)+\sum_{\alpha=1}^{N}\left(\boldsymbol{D}_{\mathbf{1} \boldsymbol{\alpha}}-\boldsymbol{D}_{\mathbf{1 1}}\right) \cdot \nabla_{r_{\alpha}} \hat{P}_{N-1 / 1}^{(0,0)},
\end{aligned}
$$

with the boundary condition

$$
\sum_{\alpha=2}^{N} n_{1 \alpha} \cdot\left(\left[U_{\alpha}-U_{1}^{(0)}\right] d^{\prime}-D_{\alpha \beta}^{r e l} \cdot \nabla_{r_{\beta}} d^{\prime}\right)=0 .
$$

For suspensions that satisfy fluctuation-dissipation, the right-hand side of Eq. (B14) is zero, and the boundary condition allows the null solution $\boldsymbol{d}^{\prime}=0$. 


\section{APPENDIX C: MICROSTRUCTURE, $Q^{\prime}=0$}

The governing equations for the microrheology problem in 3 dimensions are

$$
\begin{array}{r}
\nabla_{r} \cdot\left[U_{0} \boldsymbol{m}^{\prime}-D^{r e l} \nabla n^{\prime}\right]=\boldsymbol{M}^{U \boldsymbol{U F}} \cdot \boldsymbol{F}^{\text {ext }} \cdot \nabla n_{0}, \\
\nabla_{r} \cdot\left[U_{0} \boldsymbol{I} \frac{n^{\prime}}{3}-D^{r e l} \nabla \boldsymbol{m}^{\prime}\right]+2 D_{R} \boldsymbol{m}^{\prime}=\boldsymbol{M}^{U \boldsymbol{U F}} \cdot \boldsymbol{F}^{\text {ext }} \cdot \nabla \boldsymbol{m}_{\mathbf{0}},
\end{array}
$$

with the boundary conditions

$$
\begin{gathered}
\boldsymbol{n} \cdot\left[U_{0} \boldsymbol{m}^{\prime}-D^{r e l} \nabla n^{\prime}\right]=\boldsymbol{n} \cdot \boldsymbol{M}^{U \boldsymbol{F}} \cdot \boldsymbol{F}^{\boldsymbol{e x t}} n_{0}, r=R_{c}, \\
\boldsymbol{n} \cdot\left[U_{0} \boldsymbol{I} \frac{n^{\prime}}{3}-D^{r e l} \nabla \boldsymbol{m}^{\prime}\right]=\boldsymbol{n} \cdot \boldsymbol{M}^{U F} \cdot \boldsymbol{F}^{\boldsymbol{e x t}} \boldsymbol{m}_{\mathbf{0}}, r=R_{c}, \\
n^{\prime}, \boldsymbol{m}^{\prime} \sim \mathbf{0}, r \rightarrow \infty .
\end{gathered}
$$

The natural dimensionless groups that arise in scaling the equations are the swim Péclet number $P e_{s}=U_{0} R_{c} / D^{r e l}$, the rotational parameter $\gamma^{2}=2 D_{R} R_{c}^{2} / D^{r e l}$, and the Péclet number $P e=U^{\text {probe }} R_{c} / D^{r e l}$. In this article, $P e \ll 1$ but $P e_{s}$ and $\gamma^{2}$ may take any non-negative value.

The unit vector $\hat{\boldsymbol{u}}$ points in the direction of the applied external force (or the probe's prescribed external velocity), and the solutions for $n_{0}$ are known

$$
\frac{n_{0}}{n^{\infty}}=1+\frac{P e_{s}^{2} / 3}{\gamma^{2}+2(1+\lambda)} \frac{e^{-\lambda(r-1)}}{r},
$$

where $\lambda=\sqrt{P e_{s}^{2} / 3+\gamma^{2}}$ and the polar order is proportional to the gradient in the number density $\boldsymbol{m}_{\mathbf{0}}=P e_{s}^{-1} \nabla n_{0}$. Note that $\lambda$ is dimensionless here, but has units of inverse length in the main text. The governing equations are linear and may be solved via the same screened harmonic decomposition methods used to determine the equilibrium solution. ${ }^{28}$ The only difference here is that both governing equations are now forced by the divergence of the equilibrium advective flux, and so one must find a particular solution in addition to the homogeneous solutions found via the multipole expansion.

In $3-\mathrm{D}$, the concentration disturbance $n^{\prime}$ in terms of two undetermined coefficients is

$$
\begin{aligned}
n^{\prime}= & \hat{\boldsymbol{u}} \cdot \boldsymbol{n}\left(B_{1} \frac{1}{r^{2}}-\frac{\frac{1}{9} P e_{s}^{4} / \lambda^{4}}{2(\lambda+1)+\gamma^{2}} e^{-\lambda(r-1)}\left(\frac{\lambda}{r}+\frac{1}{r^{2}}\right)\right. \\
& -A_{1} \frac{P e_{s}}{\lambda^{2}} e^{-\lambda(r-1)}\left(\frac{\lambda}{r}+\frac{1}{r^{2}}\right)-\frac{\frac{1}{3} P e_{s}^{2} / \lambda^{2}}{2(\lambda+1)+\gamma^{2}}\left(\frac{1}{3} \frac{P e_{s}^{2}}{\lambda^{2}}+1\right) e^{-\lambda(r-1)} \\
& \left.\times\left(\frac{3}{4 \lambda^{2}} \frac{1}{r^{2}}+\frac{3}{4 \lambda} \frac{1}{r}+\frac{1}{2}\right)\right),
\end{aligned}
$$

where the coefficients $A_{1}$ and $B_{1}$ are coupled to the polar order disturbance

$$
\begin{aligned}
\boldsymbol{m}^{\prime}= & C_{1} e^{-\gamma(r-1)} \hat{\boldsymbol{u}} \cdot\left\{\frac{\boldsymbol{I}}{r}-\frac{1}{\gamma^{2}}\left[\left(3 \frac{\boldsymbol{n}}{r^{3}}-\frac{\boldsymbol{I}}{r^{3}}\right)+\gamma\left(3 \frac{\boldsymbol{n} \boldsymbol{n}}{r^{2}}-\frac{\boldsymbol{I}}{r^{2}}\right)+\gamma^{2} \frac{\boldsymbol{n} \boldsymbol{n}}{r}\right]\right\}+B_{1} \frac{1}{3} \frac{P e_{s}}{\gamma^{2}} \hat{\boldsymbol{u}} \cdot\left(3 \frac{\boldsymbol{n} \boldsymbol{n}}{r^{3}}-\frac{\boldsymbol{I}}{r^{3}}\right)-\frac{1}{P e_{s}} \frac{1}{2(\lambda+1)+\gamma^{2}} e^{-\lambda(r-1)} \\
& \times \hat{\boldsymbol{u}} \cdot\left[\left(3 \frac{\boldsymbol{n} \boldsymbol{n}}{r^{3}}-\frac{\boldsymbol{I}}{r^{3}}\right)+\lambda\left(3 \frac{\boldsymbol{n} \boldsymbol{n}}{r^{2}}-\frac{\boldsymbol{I}}{r^{2}}\right)+\lambda^{2} \frac{\boldsymbol{n} \boldsymbol{n}}{r}\right]-\frac{\frac{1}{3} P e_{s} / \lambda^{2}}{2(\lambda+1)+\gamma^{2}} e^{-\lambda(r-1)} \hat{\boldsymbol{u}} \cdot\left[\left(3 \frac{\boldsymbol{n} \boldsymbol{n}}{r^{3}}-\frac{\boldsymbol{I}}{r^{3}}\right)+\lambda\left(3 \frac{\boldsymbol{n} \boldsymbol{n}}{r^{2}}-\frac{\boldsymbol{I}}{r^{2}}\right)+\lambda^{2} \frac{\boldsymbol{n} \boldsymbol{n}}{r}\right] \\
& +\frac{1}{2(\lambda+1)+\gamma^{2}} \frac{2 \lambda^{2}-\gamma^{2}}{\lambda^{2} P e_{s}}\left(\frac{1}{3} \frac{P e_{s}^{2}}{\lambda^{2}}+1\right) e^{-\lambda(r-1)} \hat{\boldsymbol{u}} \cdot\left[\left(3 \frac{\boldsymbol{n} \boldsymbol{n}}{r^{3}}-\frac{\boldsymbol{I}}{r^{3}}\right)+\lambda\left(3 \frac{\boldsymbol{n} \boldsymbol{n}}{r^{2}}-\frac{\boldsymbol{I}}{r^{2}}\right)+\lambda^{2} \frac{\boldsymbol{n} \boldsymbol{n}}{r}\right] \\
& +\frac{1}{\lambda^{2}} A_{1} e^{-\lambda(r-1)} \hat{\boldsymbol{u}} \cdot\left[\left(3 \frac{\boldsymbol{n} \boldsymbol{n}}{r^{3}}-\frac{\boldsymbol{I}}{r^{3}}\right)+\lambda\left(3 \frac{\boldsymbol{n} \boldsymbol{n}}{r^{2}}-\frac{\boldsymbol{I}}{r^{2}}\right)+\lambda^{2} \frac{\boldsymbol{n} \boldsymbol{n}}{r}\right]+\frac{\frac{1}{3} P e_{s}}{2(\lambda+1)+\gamma^{2}}\left(\frac{1}{3} \frac{P e_{s}^{2}}{\lambda^{2}}+1\right) e^{-\lambda(r-1)} \\
& \times \hat{\boldsymbol{u}} \cdot\left[\frac{3}{4 \lambda^{2}}(\lambda r+1)\left(\frac{\boldsymbol{I}}{r^{3}}-3 \frac{\boldsymbol{n} \boldsymbol{n}}{r^{3}}\right)+\frac{1}{2}\left(\frac{\boldsymbol{I}}{r}-\frac{\boldsymbol{n} \boldsymbol{n}}{r}\right)-\lambda \boldsymbol{n n}\left(\frac{3}{4 \lambda^{2} r}+\frac{1}{2}\right)\right] .
\end{aligned}
$$

The coefficients $A_{1}, B_{1}$, and $C_{1}$ are determined by the no-flux boundary condition at particle contact. This results in a linear system of three equations that may be solved to find algebraic expressions for $n^{\prime}$

$$
\begin{aligned}
& 2 B_{1}\left(1+\frac{P e_{s}^{2}}{3 \gamma^{2}}\right)-2 C_{1} P e_{s}\left(\frac{1+\gamma}{\gamma^{2}}\right)=1-2 C_{0} \frac{(1+\lambda)}{\lambda^{4}},(\mathrm{C} 9) \\
& B_{1} \frac{P e_{s}}{\gamma^{2}}-C_{1}\left(2+\frac{3}{\gamma^{2}}+\frac{3}{\gamma}+\gamma\right)+A_{1}\left(1+\frac{3}{\lambda}+\frac{3}{\lambda^{2}}\right)=\frac{C_{0}}{\lambda^{2}}\left[\left(\frac{3}{\lambda^{2}}+\frac{3}{\lambda}+1\right)\left(1+\frac{3}{P e_{s}^{2}}-\frac{3\left(2 \lambda^{2}-\gamma^{2}\right)^{2}}{\lambda^{6} P e_{s}^{2}}\right)-\left(1+\frac{P e_{s}^{2}}{3 \lambda^{2}}\right)\left(\frac{1}{2}+\frac{9}{4 \lambda^{2}}+\frac{3}{2 \lambda}+\frac{\lambda}{2}=\frac{3(1+\lambda)}{4 \lambda}\right)\right], \\
&-B_{1} P e_{s}\left(\frac{\gamma^{2}+9}{3 \gamma^{2}}\right)+C_{1}\left(4+\frac{9}{\gamma^{2}}+\frac{9}{\gamma}+\gamma\right)-A_{1}\left(\lambda+4+\frac{9}{\lambda}+\frac{9}{\lambda^{2}}\right)+A_{1} \frac{(1+\lambda) P e_{s}^{2}}{3 \lambda^{2}}-\frac{C_{0}(1+\lambda)}{2 \lambda^{2}}-\frac{C_{0}}{\lambda^{4}}\left(2 \lambda^{2}-\gamma^{2}\right) \\
& \times\left(\frac{5}{4}+\frac{27}{4 \lambda^{2}}+\frac{9}{2 \lambda}+\frac{5 \lambda}{4}+\frac{\lambda^{2}}{2}+\frac{9(1+\lambda)}{4 \lambda}\right)-C_{0}\left(9+9 \lambda+4 \lambda^{2}+\lambda^{3}\right)\left(-\frac{1}{\lambda^{4}}-\frac{3}{\lambda^{2}\left(P e_{s}^{2}\right)}+\frac{\left(2 \lambda^{2}-\gamma^{2}\right)^{2}}{\lambda^{6}\left(\lambda^{2}-\gamma^{2}\right)}\right) \\
&=\frac{P e_{s}}{3}\left[\frac{C_{0} P e_{s}^{3}(1+\lambda)}{3 \lambda^{6}}+\frac{C_{0}\left(2 \lambda^{2}-\gamma^{2}\right)}{\lambda^{4}}\left(\frac{1}{2}+\frac{3(1+\lambda}{4 \lambda^{2}}\right)\right],
\end{aligned}
$$

where we define $C_{0}=\left(P e_{s}^{2} / 3\right) /\left(\gamma^{2}+2(1+\lambda)\right)$ for brevity. 
The analysis in $d=2$ follows precisely the same formula, but the solution will be in terms of gradients of the modified Bessel function $K_{0}(\lambda r)^{3}$

The microviscosity coefficient is determined by $n^{\prime}$

$$
\begin{aligned}
\eta^{\text {micro }}= & 2\left[B_{1}-\frac{\frac{1}{9} P e_{s}^{4} / \lambda^{4}}{2(\lambda+1)+\gamma^{2}}(\lambda+1)-A_{1} \frac{P e_{s}}{\lambda^{2}}(\lambda+1)\right. \\
& \left.-\frac{\frac{1}{3} P e_{s}^{2} / \lambda^{2}}{2(\lambda+1)+\gamma^{2}}\left(\frac{1}{3} \frac{P e_{s}^{2}}{\lambda^{2}}+1\right)\left(\frac{3}{4 \lambda^{2}}+\frac{3}{4 \lambda}+\frac{1}{2}\right)\right],
\end{aligned}
$$

where we have left the expression in terms of the coefficients $A_{1}$ and $B_{1}$ for both brevity and generality-this construction for the microstructure is valid even when the contact boundary condition is not one of no translational flux. When $P e_{s}=0, n^{\prime}=B_{1} \hat{\boldsymbol{u}} \cdot \boldsymbol{n} / r^{2}$ and $B_{1}=1 / 2$, so $\eta^{\text {micro }}=1$.

\section{APPENDIX D: EFFECTS OF CLOSURE}

Figure 3 reveals that the closure of the Smoluchowski equation seems to make a larger quantitative difference than in previous studies; ${ }^{28,36-38}$ this may be explained by simple geometric arguments. When the probe is stationary the only vector with which the swimmers may align is the probe's outward-pointing unit normal $\boldsymbol{n}$, and the polar order (whether the swimmers are moving toward or away from the probe) is the most important ordered field. The nematic order would contain terms proportional to $\boldsymbol{n} \boldsymbol{n}$ and $\boldsymbol{I}$, which do not significantly affect the kinetic boundary-layer structure, even if the probe were of some arbitrary shape. ${ }^{36}$ With the introduction of an external force (or orienting field) in a fixed direction $\hat{\boldsymbol{u}}$, the swimmers may now be swimming toward or away from the probe $\boldsymbol{m}^{\prime} \sim \hat{\boldsymbol{u}} \cdot \boldsymbol{n} \boldsymbol{n}$ or with or against the external field $\boldsymbol{m}^{\prime} \sim \hat{\boldsymbol{u}}$, as the solution in Appendix $C$ reveals. This implies that the nematic order may have terms proportional to $\hat{\boldsymbol{u}} \boldsymbol{n},(\hat{\boldsymbol{u}} \cdot \boldsymbol{n}) \boldsymbol{I}$, and $(\hat{\boldsymbol{u}} \cdot \boldsymbol{n}) \boldsymbol{n} \boldsymbol{n}$. These correlations between the external force director $\hat{\boldsymbol{u}}$ and the unit normal of the surface $\boldsymbol{n}$ must make a more significant quantitative impact in this problem. Here, we consider a test problem-the linear microrheology of an active suspension confined to an interface $(d=2)$ - and systematically investigate the effects of various closures on the computed probability distribution and its physically relevant moduli.

\section{Closures of the Smoluchowski equation}

For problems near "equilibrium"-meaning the microstructure is nearly isotropic - the most common assumption is to say that all higher order moments are isotropic

$$
\begin{gathered}
\langle\boldsymbol{q} \boldsymbol{q}\rangle=\frac{1}{d} \boldsymbol{I} n, \\
\langle\boldsymbol{q} \boldsymbol{q} \boldsymbol{q}\rangle=\frac{1}{d+2} \boldsymbol{\alpha} \cdot \boldsymbol{m}, \\
\langle\boldsymbol{q} \boldsymbol{q q} \boldsymbol{q}\rangle=\frac{1}{d(d+2)} \boldsymbol{\alpha} n+\frac{1}{2(d+4)} \boldsymbol{\kappa}: \boldsymbol{Q} \ldots,
\end{gathered}
$$

where $\boldsymbol{\alpha}$ is the fourth-order isotropic tensor and $\boldsymbol{\kappa}$ is the sixth-order isotropic tensor in $d$ dimensions. Isotropic closures are used in the main text to truncate the tensor harmonic expansion of $P$, and in previous calculations of the force on a boundary, ${ }^{37}$ as well as other works. $^{53,54}$

Isotropic closures-which amount to a truncation of the expansion of the probability distribution in tensor harmonics of $\boldsymbol{q}-$ are derived such that there can be no steady state tensorial order in the absence of spatial fluctuations: an unbounded suspension at steady state should be isotropic. For calculations of the force on a boundary, assuming isotropic nematic order $\boldsymbol{Q}=0$ correctly predicts the number density profile near the wall. This remains true in the presence of hydrodynamic interactions, as they do not substantially influence the geometry of the problem. ${ }^{38}$ From a purely mathematical perspective, we only need an accurate prediction of the zeroth moment $n$ to compute the pressure at the wall. Assuming $\boldsymbol{Q}=0$ correctly predicts the number density, but not the polar order $\boldsymbol{m}$. However, the important dynamic featurethat the polar order indicates that the swimmers move toward the wall on average-is captured with this closure. If we needed to compute a vector quantity-which would directly or indirectly depend on the polar order-we need to include $\boldsymbol{Q}$ so that the prediction of $\boldsymbol{m}$ is correct. Indeed, one could deduce that numerically accurate predictions for $\boldsymbol{Q}$ would require the calculation of $\boldsymbol{B}=\langle\boldsymbol{q q q}\rangle-\boldsymbol{\alpha} \cdot \boldsymbol{m} /(d+2)$.

One alternative approach that circumvents the need for higher moments is to explicitly include the effects of long-ranged spatial fluctuations in the equation closures. For example, one could "slave" the nematic order to the polar order

$$
\boldsymbol{Q}=-\frac{\ell / R_{c}}{2 d(d+2)}\left[\nabla \boldsymbol{m}+(\nabla \boldsymbol{m})^{\dagger}-\boldsymbol{I}(\nabla \cdot \boldsymbol{m})\right]
$$

and compare this to predictions of the microviscosity coefficient from solving the full Smoluchowski equation in position and orientation space. Here, we explicitly compare solutions of the full Smoluchowski equation with solutions of the moments expansions using closures (D1), (D2), and (D4).

\section{Full Smoluchowski equation: No closure}

Consider the motion of a probe particle through a suspension of ABPs; the dynamics are described by

$$
\frac{\partial P}{\partial t}+\nabla_{x_{P}} \cdot \boldsymbol{j}_{P}^{T}+\nabla_{x_{s}} \cdot \boldsymbol{j}_{s}^{T}+\nabla_{q_{P}} \cdot \boldsymbol{j}_{P}^{R}+\nabla_{q_{s}} \cdot \boldsymbol{j}_{s}^{R}=0,
$$

where $P\left(\boldsymbol{x}_{P}, \boldsymbol{x}_{s}, \boldsymbol{q}_{P}, \boldsymbol{q}_{s}, t\right)$ is the probability of finding the probe at position $\boldsymbol{x}_{P}$ pointing in the direction $\boldsymbol{q}_{P}$, and the swimmer at position $\boldsymbol{x}_{s}$ pointing in the direction $\boldsymbol{q}_{s}$ at time $t$. We wish to rewrite the equation in terms of a conditional probability of finding the swimmer at some point $(\boldsymbol{r}, \boldsymbol{q})$ in phase space, given that the probe is at the point $(\boldsymbol{z}, \boldsymbol{p})$. Transforming the position-space equations into a relative coordinate frame is simple vector addition $-\boldsymbol{z}^{\prime} \equiv \boldsymbol{x}_{P}$, $\boldsymbol{r}^{\prime} \equiv \boldsymbol{x}_{s}-\boldsymbol{x}_{P}$-but moving into a frame where the swimmer rotates relative to the probe requires a matrix operator $\boldsymbol{R}\left(\boldsymbol{q}_{P}\right)$ that rotates these position vectors into the appropriate frame: $\boldsymbol{z}=\boldsymbol{z}^{\prime}, \boldsymbol{r}=\boldsymbol{R} \cdot \boldsymbol{r}^{\prime}$, $\boldsymbol{p}=\boldsymbol{q}_{P}, \boldsymbol{q}=\boldsymbol{R} \cdot \boldsymbol{q}_{s}$. In the microrheology problem, the direction of the probe velocity (or external force) $\hat{\boldsymbol{u}}$ determines the orientation of the probe, so we require $\boldsymbol{R}\left(\boldsymbol{q}_{P}\right) \cdot \boldsymbol{q}_{\boldsymbol{P}}=\hat{\boldsymbol{u}} .^{55}$ For $d=2$, this rotation is parameterized only by the polar angle. In $3-\mathrm{D}$, the rotation matrix would be defined in the same manner, but we would require both a 
polar and azimuthal coordinate to parametrize the rotation. The 3-D rotation is not unique.

For simplicity, we will assume that we are working in twodimensions, and that the probe is moving in the $x$-direction $\hat{\boldsymbol{u}}=\boldsymbol{e}_{x}$ (see Fig. 7). The unit normal of the probe-the unit vector pointing along $\boldsymbol{r}^{\prime}$-is thus given by $\boldsymbol{n}=\cos \theta \boldsymbol{e}_{x}+\sin \theta \boldsymbol{e}_{y}$. We can assume that $\boldsymbol{z}^{\prime}$ is at the origin. The orientation of the swimmer is $\boldsymbol{q}_{s}=\cos \psi \boldsymbol{e}_{x}+\sin \psi \boldsymbol{e}_{y}$. The fluxes of each particle are now as follows:

$$
\begin{aligned}
\boldsymbol{j}_{\boldsymbol{P}}^{\boldsymbol{T}} & =U^{\text {probe }} \boldsymbol{e}_{x} P-D_{P} \nabla_{z}^{\prime} P+D_{P} \nabla_{r}^{\prime} P, \\
\boldsymbol{j}_{\boldsymbol{s}}^{\boldsymbol{T}} & =U_{0} \boldsymbol{q}_{s} P-D_{T} \nabla_{r}^{\prime} P, \\
\boldsymbol{j}_{\boldsymbol{P}}^{\boldsymbol{R}} & =-D_{R} \nabla_{q_{P}} P, \\
\boldsymbol{j}_{\boldsymbol{s}}^{\boldsymbol{R}} & =-\frac{1}{\tau_{R}} \nabla_{q_{s}} P,
\end{aligned}
$$

where $D_{P}$ is the thermal translational diffusivity of the probe, and $D_{R}$ is the probe's rotational diffusivity. The speed of the swimmers is $U_{0}$, their reorientation time is $\tau_{R}$, and their translational diffusivity is $D_{T}$. Here, we have chosen to represent changes in reorientation by gradients with respect to $\boldsymbol{q}_{P}$ and $\boldsymbol{q}_{s}$; Brenner showed that $\nabla_{R}^{2}=\nabla_{q}^{2}$, so either representation is equivalent in the final equation. ${ }^{56}$ For the microviscosity, we are not concerned with fluctuations in the absolute position (and orientation) of the probe-more care is needed when computing the effects of activity on the effective long-time self-diffusivity of the probe. All rotations are isometries and members of the special orthogonal group $S O(d)$; consequently $\boldsymbol{R}^{-1}=\boldsymbol{R}^{\dagger}$ and the dot product is invariant under rotation. Thus, we circumvent the need to write the flux expressions in rotating coordinates, and may write down the final, scalar equation in the coordinates $r, \theta$, and $\beta \equiv \psi-\theta$

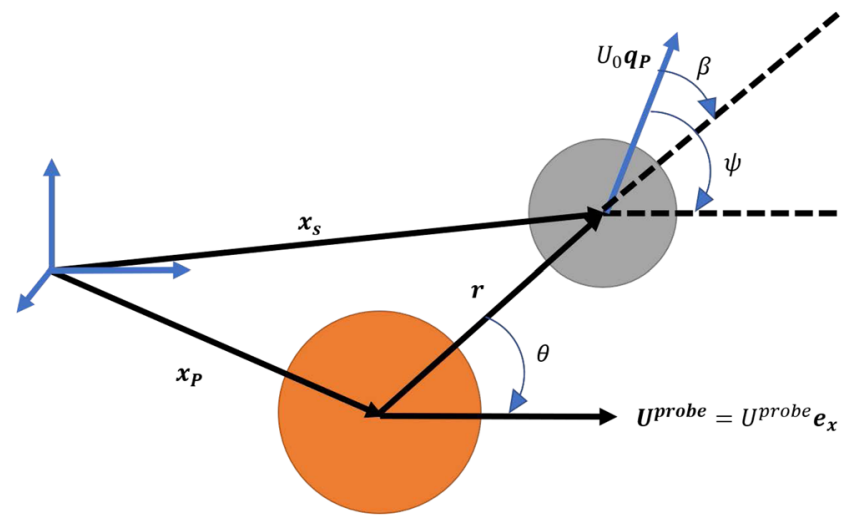

FIG. 7. Sketch of the probe-swimmer coordinate system. The probe has a position $\boldsymbol{x}_{P}$ and orientation $\boldsymbol{e}_{\boldsymbol{x}}$ in the fixed coordinate frame. The swimmer has a position $\boldsymbol{x}_{s}$ and orientation $\boldsymbol{q}_{s}$ (parametrized by the angle $\psi$ ) in the fixed frame. The relative separation between the particles is $r$, and the polar angle of the swimmer's position with respect to the probe is $\theta$. The orientation of the swimmer relative to the probe is parametrized by the angle $\beta=\psi-\theta$.

$$
\begin{aligned}
\frac{\partial P}{\partial t}+ & U_{0}\left(\cos \beta \frac{\partial P}{\partial r}-\frac{\sin \beta}{r} \frac{\partial P}{\partial \beta}+\frac{\sin \beta}{r} \frac{\partial P}{\partial \theta}\right) \\
& -U^{\text {probe }}\left(\cos \theta \frac{\partial P}{\partial r}-\frac{\sin \theta}{r} \frac{\partial P}{\partial \theta}+\frac{\sin \theta}{r} \frac{\partial P}{\partial \beta}\right) \\
& -D^{r e l}\left(\frac{\partial^{2} P}{\partial r^{2}}+\frac{1}{r} \frac{\partial P}{\partial r}+\frac{1}{r^{2}} \frac{\partial^{2} P}{\partial \beta^{2}}+\frac{1}{r^{2}} \frac{\partial^{2} P}{\partial \theta^{2}}\right) \\
& -\left(\tau_{R}^{-1}+D_{R}\right) \frac{\partial^{2} P}{\partial \beta^{2}}-D_{R} \frac{\partial^{2} P}{\partial \theta^{2}}=0 .
\end{aligned}
$$

The derivatives with respect to $\beta$ are lost if one does not carefully consider the transformation to a frame rotating with the probe.

When $U^{\text {probe }} \equiv 0$ there can be no dependence on the angle $\theta$, as there is no symmetry breaking without directed motion. For small perturbations away from equilibrium, we know that the probability distribution should take the form $P=P_{0}(r, \beta)+P e \cos \theta f(r, \beta)$, where $P e=U^{\text {probe }} R_{c} / D^{\text {rel }}$. So we now do a moments expansion in $\theta$ rather than in $\beta$. Indeed, this is most appropriate when we allow the magnitude of activity to be arbitrary, but consider only small perturbations due to external forces. follows:

The governing equations in the absence of probe motion are as

$$
\begin{array}{rr}
P e_{s}\left(\cos \beta \frac{\partial P_{0}}{\partial r}-\frac{\sin \beta}{r} \frac{\partial P_{0}}{\partial \beta}\right)-\frac{\partial^{2} P_{0}}{\partial r^{2}}-\frac{1}{r} \frac{\partial P_{0}}{\partial r}-\left(\frac{1}{r^{2}}+\gamma^{2}\right) \frac{\partial^{2} P_{0}}{\partial \beta^{2}}=0, & (\mathrm{D} 11) \\
P e_{s} \cos \beta P_{0}-\frac{\partial P_{0}}{\partial r}=0, r=1, & \text { (D12) } \\
P_{0} \sim n^{\infty} / 2 \pi, r \rightarrow \infty, & \text { (D13) }
\end{array}
$$

where $P e_{s}=U_{0} R_{c} / D^{r e l}, \gamma^{2}=R_{c}^{2} / D^{r e l}\left(\tau_{R}-D_{R}^{-1}\right)$. The $O(P e)$ equations are

$$
\begin{gathered}
P e_{s}\left(\cos \beta \frac{\partial f}{\partial r}-\frac{\sin \beta}{r} \frac{\partial f}{\partial \beta}\right)-\frac{\partial P_{0}}{\partial r}-\frac{\partial^{2} f}{\partial r^{2}}-\frac{1}{r} \frac{\partial f}{\partial r}+\frac{f}{r^{2}} \\
-\left(\frac{1}{r^{2}}+\gamma^{2}\right) \frac{\partial^{2} f}{\partial \beta^{2}}=0, \\
P e_{s} \cos \beta f-P_{0}-\frac{\partial f}{\partial r}=0, r=1, \\
P_{1} \sim 0, r \rightarrow \infty .
\end{gathered}
$$

The governing equation for $f$ is obtained by multiplying the full $O(P e)$ equation by $\cos \theta$ and integrating with respect to $\theta$. Thus, the microviscosity coefficient will simply be $\eta^{\text {micro }}=\int f(1, \beta) d \beta$.

\section{Finite-element results, $d=2$}

Finite-element solutions for $P_{0}(r, \beta)$ and $f(r, \beta)$ are done in FreeFEM,$++{ }^{57}$ which uses a standard Galerkin P2-FEM method with adaptive mesh refinement. From $P_{0}$ and $f$, we can directly compute the various moments of $\mathrm{P}$ as a Fourier series expansion

$$
P(r, \theta, \beta)=\sum_{i=0}^{\infty} a_{i}(r, \theta) \cos (i \beta)+b_{i}(r, \theta) \sin (i \beta),
$$




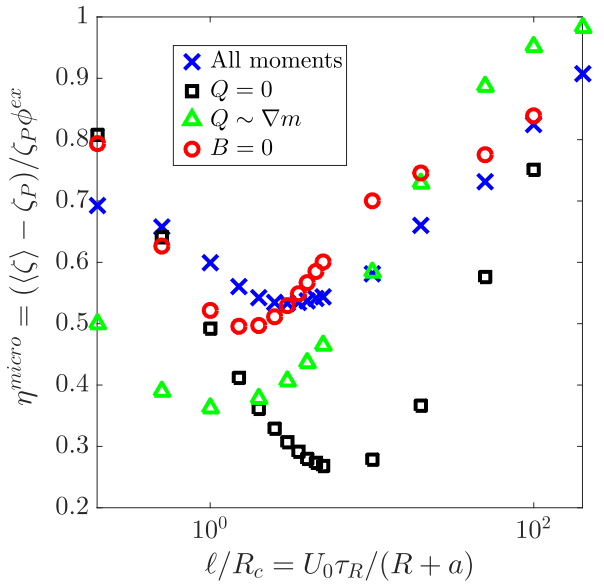

FIG. 8. Microviscosity coefficient $(d=2)$ as a function of $\ell / R_{c}$ at $\ell / \delta=10$ for various closures of the moments expansion of $P$. The squares and crosses are from finite difference solutions of the 2-D governing equations in MATLAB, and the circles are from finite element simulations of the full Smoluchowski equation in FreeFEM++. ${ }^{5}$

where

$$
\begin{aligned}
& a_{i}(r, \theta)=\frac{1}{\pi} \int P \cos (i \beta) d \beta, \\
& b_{i}(r, \theta)=\frac{1}{\pi} \int P \sin (i \beta) d \beta,
\end{aligned}
$$

and $i$ is some positive integer (in the special case of $a_{0}$, the coefficient of the integral is $1 / 2 \pi$ ). These coefficients in the series expansions are the moments in the field equations introduced previously: $n=a_{0}$, $\boldsymbol{m}=a_{1} \boldsymbol{e}_{r}+b_{1} \boldsymbol{e}_{\theta}$, etc.

In Fig. 8, we plot the microviscosity coefficient as a function of $\ell / R_{c}$ for various closures. Even the simplest closure $Q=0$ correctly captures the limiting behavior for $\ell / R_{c} \rightarrow 0$ and $\ell / R_{c} \rightarrow \infty$, and the qualitative shape of the distribution. Allowing for fluctuations in nematic order by slaving $\boldsymbol{Q}$ to $\nabla \boldsymbol{m}$ improves the quantitative accuracy, but $\boldsymbol{B}=0$ provides the best quantitative match to the exact solution.

All closures capture the trend with respect to $\ell / R_{c}$. We can check the limiting behaviors in the microviscosity coefficient from a boundary-layer analysis. Equations (D11) and (D14) can be rewritten in terms of a boundary-layer coordinate $Y=P e_{s}(r-1)$ when $P e_{s} \gg 1$. If $\gamma / P e_{s} \ll 1\left(\ell / R_{c} \gg 1\right)$, then one finds that the microviscosity coefficient approaches 1 as $P e_{s} \rightarrow \infty$. Near the viscosity minimum, we must retain terms $\sim O\left(\gamma / P e_{s}\right)^{2}$ to capture the minimum properly; unfortunately, the boundary-layer analysis breaks down here. We conclude that $\boldsymbol{B}=0$ is the best closure to use in the microrheology problem, and indeed may be the best closure when computing any vector quantity in active suspensions.

\section{REFERENCES}

${ }^{1}$ R. Kubo, Rep. Prog. Phys. 29, 255 (1966).

${ }^{2}$ H. B. Callen and T. A. Welton, Phys. Rev. 83, 34 (1951).

${ }^{3}$ M. Doi, Soft Matter Physics (Oxford University Press, Oxford, UK, 2013).

${ }^{4}$ J. F. Brady, J. Fluid Mech. 272, 109 (1994).
${ }^{5}$ S. Ramaswamy, Annu. Rev. Condens. Matter Phys. 1, 323 (2010); e-print arXiv:1004.1933.

${ }^{6}$ M. C. Marchetti, J. F. Joanny, S. Ramaswamy, T. B. Liverpool, J. Prost, M. Rao, and R. A. Simha, Rev. Mod. Phys. 85, 1143 (2013); e-print arXiv:1207.2929.

${ }^{7}$ S. C. Takatori and J. F. Brady, Phys. Rev. E 91, 032117 (2015); e-print arXiv:1411.5776.

${ }^{8}$ A. P. Solon, M. E. Cates, and J. Tailleur, Eur. Phys. J.: Spec. Top. 224, 1231 (2015).

${ }^{9}$ S. C. Takatori and J. F. Brady, Soft Matter 11, 7920 (2015); e-print arXiv:1011.1669v3.

${ }^{10}$ X.-L. Wu and A. Libchaber, Phys. Rev. Lett. 84, 3017 (2000).

${ }^{11}$ M. J. Kim and K. S. Breuer, Phys. Fluids 16, L78 (2004).

${ }^{12}$ G. Miño, T. E. Mallouk, T. Darnige, M. Hoyos, J. Dauchet, J. Dunstan, R. Soto, Y. Wang, A. Rousselet, and É. Clément, Phys. Rev. Lett. 106, 048102 (2011); e-print arXiv:1012.4624.

${ }^{13}$ A. Jepson, V. A. Martinez, J. Schwarz-Linek, A. Morozov, and W. C. K. Poon, Phys. Rev. E 88, 041002 (2013); e-print arXiv:1307.1274.

${ }^{14}$ G. Miño, J. Dunstan, A. Rousselet, É. Clément, and R. Soto, J. Fluid Mech. 729, 423 (2013); e-print arXiv:1210.7704.

${ }^{15}$ J.-L. Thiffeault and S. Childress, Phys. Lett. A 374, 3487 (2010); e-print arXiv:0911.5511.

${ }^{16}$ D. O. Pushkin and J. M. Yeomans, Phys. Rev. Lett. 111, 188101 (2013); e-print arXiv:1307.6025v1.

${ }^{17}$ A. Morozov and D. Marenduzzo, Soft Matter 10, 2748 (2014); e-print arXiv:1308.3387v1.

${ }^{18}$ R. Jeanneret, D. O. Pushkin, V. Kantsler, and M. Polin, Nat. Commun. 7, 12518 (2016); e-print arXiv:1602.01666.

${ }^{19}$ Y. Hatwalne, S. Ramaswamy, M. Rao, and R. A. Simha, Phys. Rev. Lett. 92, 118101 (2004); e-print arXiv:0308529 [cond-mat].

${ }^{20}$ B. M. Haines, I. S. Aronson, L. V. Berlyand, and D. A. Karpeev, Phys. Biol. 5, 046003 (2008).

${ }^{21}$ A. Sokolov and I. S. Aranson, Phys. Rev. Lett. 103, 148101 (2009).

${ }^{22}$ D. Saintillan, Phys. Rev. E - Stat. Nonlinear, Soft Matter Phys. 81, 056307 (2010).

${ }^{23}$ G. Foffano, J. S. Lintuvuori, A. N. Morozov, K. Stratford, M. E. Cates, and D. Marenduzzo, Eur. Phys. J. E 35, 98 (2012); e-print arXiv:1211.3544.

${ }^{24}$ H. M. López, J. Gachelin, C. Douarche, H. Auradou, and É. Clément, Phys. Rev. Lett. 115, 028301 (2015).

${ }^{25}$ D. T. N. Chen, A. W. Lau, L. A. Hough, M. F. Islam, M. Goulian, T. C. Lubensky, and A. G. Yodh, Phys. Rev. Lett. 99, 148302 (2007); e-print arXiv:0709.1465.

${ }^{26}$ T. V. Kasyap, D. L. Koch, and M. Wu, Phys. Fluids 26, 081901 (2014).

${ }^{27}$ A. E. Patteson, A. Gopinath, P. K. Purohit, and P. E. Arratia, Soft Matter 12, 2365 (2016).

${ }^{28}$ E. W. Burkholder and J. F. Brady, Phys. Rev. E 95, 052605 (2017); e-print arXiv:1703.10554.

${ }^{29}$ G. Foffano, J. S. Lintuvuori, K. Stratford, M. E. Cates, and D. Marenduzzo, Phys. Rev. Lett. 109, 028103 (2012); e-print arXiv:1204.1279.

${ }^{30}$ C. Reichhardt and C. J. O. Reichhardt, Phys. Rev. E 91, 032313 (2015); e-print arXiv:1409.1586.

${ }^{31}$ S. Kim and S. J. Karilla, Microhydrodynamics: Principles and Selected Applications (Dover Publications, 1991).

${ }^{32}$ R. N. Zia and J. F. Brady, J. Fluid Mech. 658, 188 (2010).

${ }^{33}$ J. F. Brady and J. F. Morris, J. Fluid Mech. 312, 223 (1996).

${ }^{34}$ F. Smallenburg and H. Löwen, Phys. Rev. E 92, 032304 (2015); e-print arXiv:1504.05080.

${ }^{35}$ Y. Fily, A. Baskaran, and M. Hagan, Soft Matter 10, 5609 (2014).

${ }^{36}$ W. Yan and J. F. Brady, Soft Matter 14, 279 (2017); e-print arXiv:1711.01450.

${ }^{37}$ W. Yan and J. F. Brady, J. Fluid Mech. 785, R1 (2015); e-print arXiv:1510.07731.

${ }^{38}$ E. W. Burkholder and J. F. Brady, Soft Matter 14, 3581 (2018).

${ }^{39}$ D. Saintillan and M. J. Shelley, in Complex Fluids in Biological Systems, edited by S. Spagnolie (Springer, New York, 2015), Chap. 9, pp. 319-355.

${ }^{40}$ D. Saintillan and M. J. Shelley, C. R. Phys. 14, 497 (2013).

${ }^{41}$ E. W. Burkholder and J. F. Brady, "Active and nonlinear microrheology of active colloidal dispersions" (unpublished).

${ }^{42}$ A. S. Khair and J. F. Brady, J. Fluid Mech. 557, 73 (2006). 
${ }^{43}$ T. M. Squires and J. F. Brady, Phys. Fluids 17, 073101 (2005).

${ }^{44}$ S. C. Takatori and J. F. Brady, Phys. Rev. Lett. 118, 018003 (2017).

${ }^{45}$ A. Einstein, Ann. Phys. 324, 289 (1906).

${ }^{46}$ For simplicity it is assumed that the relative rotations can be written in this manner. Indeed, this is a true statement in 2-D (see Appendix D), though it is not as simple in $3-\mathrm{D}^{5}$

${ }^{47}$ One can imagine allowing the orientation coordinate to be unbounded by defining it on a Riemann surface without any branch points. Alternatively, one could take a Finite Fourier Transform with respect to $\boldsymbol{q}_{1}$. The details are not important for the discussion here.

${ }^{48}$ W. Sutherland, London, Edinburgh, Dublin Philos. Mag. J. Sci. 9, 781 (1905).

${ }^{49}$ M. von Smoluchowski, Ann. Phys. 326, 756 (1906); e-print arXiv:9811186 [arXiv:cond-mat].
${ }^{50}$ H. Brenner, Chem. Eng. Commun. 18, 355 (1982).

${ }^{51}$ This is similar to Whitehead's paradox for small (but finite) Reynolds number $(R e)$ corrections tot Stokes drag law-the inertial terms in the NavierStokes equations are only small within a distance of $1 / R e$ of the particle surface.

${ }^{52}$ J. M. Rallison and D. E. J. Hinch, J. Fluid Mech. 167, 131 (1986).

${ }^{53}$ A. Baskaran and M. C. Marchetti, Proc. Natl. Acad. Sci. U. S. A. 106, 15567 (2009).

${ }^{\mathbf{5 4}}$ T. Brotto, J. B. Caussin, E. Lauga, and D. Bartolo, Phys. Rev. Lett. 110, 038101 (2013); e-print arXiv:1208.6448.

${ }^{55}$ M. Gruber, "Active microrheology of ellipsoidal probes" (unpublished).

${ }^{56}$ H. Brenner, J. Colloid Interface Sci. 32, 141 (1970).

${ }^{57}$ F. Hecht, O. Pironneau, J. Morice, A. Le Hyaric, K. Ohtsuka, and P. Jolivet, FreeFem++, 2017. 